\title{
BORDEAUX VIVA WINTER SCHOOL - XXXIII LIAC MEETING
}

\section{November to 1 December | 29 Novembro a 1 Dezembro}

\section{Program}

29 Novembrer | 29 de Novembro

Reception of participant Winter School Meeting

Winter School meeting (organizers: A. Bikfalvi \& J. Badaut) Presentation of students - What should be achieved in this winter school Opening meeting : Thierry Couffinhal (VIVA action, Director) \& Michel Spina (LIAC President)

CLINICAL AND EPIDEMIOLOGIC ASPECT OF VASCULAR AGING (chairmen: C. Tzourio \& L. Monteiro)

Early vascular aging - P.M. NILSSON, Malmö - Sweden

Neurovascular epidemiology of aging - C. TZOURIO, Bordeaux

Cardiovascular epidemiology of aging - P. BOUTOUYRIE, Paris

Forecasted trends in disability and life expectancy - S. AHMADI-ABHARI, Liverpool - UK

Neurovascular genetic epidemiology - S. DEBETTE, Bordeaux

Vascular and thrombosis genetic epidemiology - D. TREGOUET, Paris

SELECTED ORAL PRESENTATION (Chairmen: S. Debette \& J. Badaut)

- Mitochondrial function regulates vascular aging in mice - K. FOOTE, Cambridge - UK

- Structural imaging of the vascular wall - S. ALMAGRO, Reims

- Numerical assessment and comparison of pulse wave velocity methods aiming at measuring aortic stiffness - H. OBEID, Paris

- Long-term trajectories of cardiometabolic risk factors in prodromal dementia: the Three-City Study - M. WAGNER, Bordeaux

EVENING PHILOSOPHICAL CONFERENCE ANTONIO-MARIO TAMBURRO (chairman: M. Spina)

When does the vascular system age and when is there a disease? Conceptual and theoretical issues - M. LEMOINE, Tours

30 Novembrer | 30 de Novembro

PATHOPHYSIOLOGIC FEATURES OF VASCULAR AGING (Chairmen: J.F. Arnal \& M. Formato)

From physiological aging to pathological aging - J.B. MICHEL, Paris

Physiological models to study the human microcirculation - effects of hyperoxia - L. MONTEIRO-RODRIGUES, Lisbon - Portugal

Murine models for investigating vascular ageing - G. FAURY, Grenoble

New insights into vascular biology by single cell RNA sequencing - M. VANLANDEWYCK, Stockholm - Sweden

Thrombosis in aging - C. JAMES, Bordeaux

Retinal vascular imaging - C. HELMER, Bordeaux

Lunch of Winter School Meeting

Winter School meeting (organizers: A. Bikfalvi \& J. Badaut) Meet the experts - Round table with experts

MATRIX STRUCTURAL AND MECHANICAL STUDIES (Chairmen: I. Brunet \& P. Dufourcq)

Model of pseudoxanthoma elasticum - Vascular calcification and aging, from gene to bedside - L. MARTIN, G. LEFTHERIOTIS, Angers

Vascular aging: structure and dynamics of constitutive macromolecules and sub-fragments - V. SAMOUILLAN, Toulouse

Tubular scaffolds for applications of vascular tissue engineering - V. LA CARRUBBA, Palermo - Italy

Bioprosthetic heart valve aging deterioration and age-related benefits - M. SPINA, Padova - Italy

Elastin modification during vascular aging and pathophysiological consequences - L. DUCA, Reims

\section{SELECTED ORAL PRESENTATION (Chairmen: M. Laffargue \& D. Henrion)}

- MicroRNA expressed in progenitor cells circulating in peripheral blood as prospective biomarkers of agerelated macular degeneration (AMD) in its dry and wet form - preliminary data - M. KAWA, Poland

- Molecular, conformational and thermal characterization of ventricular Remodeling in a pig model of tachycardia-induced cardiomyopathy - V.

LLORENTE CORTES, Barcelona - Spain

- Valvular calcification: implication of the Semicarbazide-Sensitive Amine Oxidase (SSAO)? - N. MERCIER, Nancy 


\section{Program (cont.)}

- Vascular calcification during chronic kidney disease: role of the RAGE/Cathepsin S/elastin peptides axis - A. WAHART, Reims

VIVA EVENING CONFERENCE (Chairman: T. Couffinhal)

A short history of the vessel - A. BIKFALVI, Bordeaux

1 December 1 de Dezembro

CELL BIOLOGY AND SIGNALING (Chairwomen: C. Duplàa \& V. Llorrente Cortes) SELECTED ORAL PRESENTATION

- Targeting Connexin40 expression or function reduces angiogenesis in the developing mouse retina - F. ALONSO, Bordeaux

- Imatinib inhibits hypoxia-induced MMP-9 overexpression and activation through impairment of LRP1 and Pyk2 phosphorylation in human coronary vascular smooth muscle cells - A. BENITEZ, Barcelona - Spain

- NRP1 supresses TGF $\beta$-dependent ERK1/2 activation in endothelial cells - A. LAMPROPOULOU, London - UK

- The elastin receptor complex interacts with CD36 through NEU1 and regulates oxidized LDL uptake in macrophages: potential implication in the proatherogenic effects of elastin-derived peptides - P. MAURICE, Reims

- Ubiad1 promotes tumor angiogenesis by inhibiting dephosphorylation of vegfr2 in a redox dependent manner - R. SEWDUTH, Belgium

Cell senescence and telomere - E. GILSON, Nice

Estrogens as vascular anti-ageing hormones? Experimental and clinical evidences and uncertainties - J.F. ARNAL, Toulouse

Arteriolar and microcirculation aging - D. HENRION, Angers

Oxydation and inflammation in Atherosclerosis - A. LEPEDDA, Italy

Winter School Meeting (organizers: A. Bikfalvi \& J. Badaut) Highlights and major topics identified by the students, group proposals (3 per group) for fictive/future projects and presentation

OPEN SESSION (Chairwomen: M.A. Renault \& J. Lods)

- Endothelial cell dysfunction is a new potential therapeutic target for the treatment of critical limb ischemia - C. CARADU, Pessac

- Podosomes Mediate Basement Membrane Collagen-IV Proteolysis During Sprouting Angiogenesis - E. GENOT, Bordeaux

-How plant food bioactives may have benefits for vascular health? The case of curcumin, L.E. MONFOULET, Lille

Closing meeting: Thierry Couffinhal \& Michel Spina 


\section{Table des matières}

\section{CLINICAL AND EPIDEMIOLOGIC ASPECT OF VASCULAR AGING}

Early vascular aging

Neurovascular epidemiology of aging

Cardiovascular epidemiology of aging

Forecasted trends in disability and life expectancy

Neurovascular genetic epidemiology

Vascular and thrombosis genetic epidemiology

\section{EVENING PHILOSOPHICAL CONFERENCE ANTONIO-MARIO TAMBURRO}

When does the vascular system age and when is there a disease? Conceptual and theoretical issues

PATHOPHYSIOLOGIC FEATURES OF VASCULAR AGING

From physiological aging to pathological aging

Physiological models to study the human microcirculation - effects of hypeoxia

Murine models for investigating vascular ageing

New insights into vascular biology by single cell RNA sequencing

Thrombosis in aging

Retinal vascular imaging

\section{MATRIX STRUCTURAL AND MECHANICAL STUDIES}

Model of pseudoxanthoma elsticum - Vascular calcification and aging, from gene to bedside

Vascular aging: structure and dynamics of constitutive macromolecules and sub-fragments

Tubular scaffolds for applications of vascular tissue engineering

Bioprosthetic heart aging détérioration and age-related benefits

Elastin modification during vascular aging and pathophysiological consequences

\section{VIVA EVENING CONFERENCE}

A short history of the vessel

\section{CELL BIOLOGY AND SIGNALING}

Cell senescence and telomere

Estrogens as vascular anti-ageing hormones? Expérimental and clinical evidences and uncertainties

Arteriolar and microcirculation agin

Oxidation and inflammation in atheriosclerosis

\section{SELECTED ORAL PRESENTATION}

Mitochondrial function regulates vascular aging in mice

Structural imaging of the vascular wal

Numerical assessment and comparison of pulse wave velocity methods aiming at measuring aortic stiffness

Long-term trajectorie of cardiometabolic risk factors in prodromal dementia: the Three-City

MicroRNA expressed in progenitor cells circulating in peripheral blood as prospective biomarkers of age related degeneration (AMD) in its dry and wet form - preliminary data

Molecular, conformational and thermal characterization pf ventricular remodeling in a pig model of tachycardia-induced cardiomyopathy

Valvular calcification: implication if the Semicarbazide-Sensitive Amine Oxidaxe (SSAO)?

Vascular calcification during chronic kidney disease: role of the RAGE/Capthepsin S/elastin peptides axis

Targeting Connexin40 expression or function reduces angiogenesis in the developing mouse retina

Imatinib inhibits hypoxia-induced MMP- overexpression and activation through impairment of LRP1 and Pyk2 phosphorylation in human cprpnary vascular smooth musle cells

NRP1 supresses TGFB-dependent ERK1/2 activation in endothelial cells

The elastin receptor complex interacts with CD36 through NEU1 and regulates oxidized LDL uptake in macrophages : potential implication in the proatherogenic effects of elastin-derived peptides

Ubiad1 promotes tumor angiogenesis by inhibiting dephosphorylation of vegfr2 in a redox dependent manner

\section{OPEN SESSION}

Endothelial cell dysfunction is a new potential therapeutic target for the treatment of critical limb ischemia

Podosomes mediate basement membrane collagen-IV proteolysis during sprouting angiogenesis

How plant foot bioactives may have benefits for vascular health? The case of curcumin 


\title{
CLINICAL AND EPIDEMIOLOGIC ASPECT OF VASCULAR AGING
}

Early Vascular Ageing (EVA) in translation: from cell senescence to clinical implications

Peter M Nilsson, $\mathrm{MD}, \mathrm{PhD}^{* 1}$

${ }_{1}$ Professor of Clinical Cardiovascular Research, Lund University, Department of Clinical Sciences, Skane ovascular Research, Lund University, Departmen
University Hospital, S-205 02 Malmo, Sweden

$$
\text { * Intervenant }
$$

Already Thomas Sydenham (1624-1689) told that "A man is as old as his arteries" The ageing process of the vasculature has increasingly been studied over the last 25 years. New data on the stiffening of arteries (arteriosclerosis) has been added to long-term studies on atherosclerosis. It is believed that arteriosclerosis starts early in life, based on fetal programming of the elastin content of the arterial media, as well as of the vasculature and capillaries. This will impact on the morphological changes of large elastic arteries and centra hemodynamic regulation. Later on the process of atherosclerosis, as being more proximal to clinical cardiovascular events, will play an important role while the two processes will be more and more mixed and jointly increase the risk. of the arterial media and a relative increase of the collagen content as well as more pronounced collagen linkages. In addition there is a mechanism called mechanotransduction that will impact on the structure of the extra-cellula matrix in relation to increased tension of the arterial wall, i.e. following increased blood pressure. This will also influence vascular smooth muscle cells (VSMC) to undergo changes as the VSMC contractile phenotype is associated with an increased arterial stiffness. This can take place both in the arterial media and the adventitia [1] In addition, some specific processes related to the adventitia may further increase stiffness. When the vascularization of the arterial wall via vasa vasonm is inpaired, as follow therapy, this has boen shown to increase arterial stiffnes.

glucose metabolism. Finally, the existence of perivasculated to both increased arterial stiffness and impaired inflammation via secretion of vasoactive cytokines. This will also influence arterial stiffness by reducing endothelial function to vasodilate.

Based on epidemiological evidence from the Malmo Diet Cancer Cohort it has been shown that increased carotidfemoral pulse wave velocity (c-f PWV) is independently associated with markers of impaired glucose metabolism [2], even adjusted for hemodynamic factors and risk factors for atherosclerosis (LDL cholesterol, smoking, renal - a different risk factor cluster the the more trational cluster asocited with atherosclerosis (hypertencion, smoking, hyperlipidaemia).

We have also found that c-f PWV is associated with a family history of cardiometabolic disease [3], impaire cognition [4], and is predictive of type 2 diabetes [5] and all-cause mortality, even adjusted for known risk factors/markers and family history of cardiometabolic disease (abstract, ARTERY 2017, Pisa, Italy).

New drugs are being developed for vascular protection and the reduction of arterial stiffiness. One promising experimental drug, currently in human Phase-1 studies, is the selective Angiotensin-II (AT2) agonist compound 21 (C21). In one animal study it was shown that C21 could reduce PWV in L-NAME induced hypertension without
interfering with blood pressure [6].

In summary, early vascular ageing (EVA) has inspired research to better understand the morphological changes of the a predictor not only of cardiovascular events, independent of traditional risk factors, but also of type 2 diabetes an total mortality. This shows that arterial stiffness is a marker of biological ageing in general and should be the focus for prevention and testing of new drugs for vascular protection such as C21, besides the efforts to reduce the burden of atherosclerosis, plaque formation and risk of cardiovascular event.

References
1. Lacolley P, Regnault V, Segers P, Laurent S. Vascular Smooth Muscle Cells and Arterial Stiffening: Relevance in Development, Aging, and Disease. Physiol Rev. 2017;97:1555-1617.

M, Ostling G, Persson M, Engström G, Melander O, Nilsson PM. Non-hemodynamic predictors of arterial stiffness after 17 years of follow-up: the Malmö Diet and Cancer study. J Hypertens. 2015;33:957-65.

3. Fatehali AA, Gottsäter M, Nilsson PM. Family history of cardiometabolic diseases and its association with arterial stiffness in the Malmö Diet Cancer cohort. J Hypertens. 2017;35:2262-2267.

4. Nilsson ED, Elmståhl S, Minthon L, Nilsson PM, Pihlsgård M, Tufvesson E, Nägga K. Nonlinear association between pulse wave velocity äd cognthe function. a population-based study. J Hypertens. 2014,32.2152-7. 5. Mwies 6. Paulis L, Becker ST, Lucht K, Schwengel K, Slavic S, Kaschina E, Thöne-Reineke C, Dahlöf B, Baulmann J, Unger T, Steckelings UM. Direct angiotensin II type 2 receptor stimulation in N $\omega$-nitro- L-arginine-methyl esterinduced hypertension: the effect on pulse wave velocity and aortic remodeling. Hypertension. 2012;59:485-92. 7. Nilsson PM, Boutouyrie P, Cunha P, Kotsis V, Narkiewicz K, Parati G, Rietzschel E, Scuteri A, Laurent S. Early vascular ageing in translation: from laboratory investigations to clinical applications in cardiovascular prevention. $J$.
Hypertens. 2013;31:1517-26.

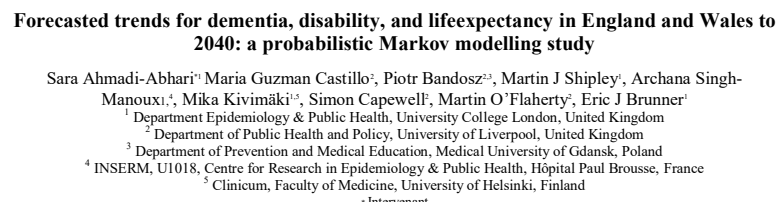

Forecasted trends for dementia, disability, and lifeexpectancy in England and Wales to 2040: a probabilistic Markov modelling study

Sara Ahmadi-Abhari' Maria Guzman Castillo ${ }^{2}$, Piotr Bandosz ${ }^{33}$, Martin J Shipley', Archana Singh-

Manouxı,4, Mika Kivimäki's, Simon Capewell', Martin O'Flaherty', Eric J Brunner

'Department Epidemiology \& Public Health, University College London, United Kingdom
2 'Department of Public Health and Policy, University of Liverpool, United Kingdom

${ }^{3}$ Department of Prevention and Medical Education, Medical University of Gdansk, Poland
INSERM, U1018, Centre for Research in Epidemiology \& Public Health, Hôpital Paul Brousse, France

SClinicum, Faculty of Medicine, University of Helsinki, Finlan

Background: As life-expectancy rises, dementia and disability are expected to impose increasing societal and healthcare burden. Forecasts for

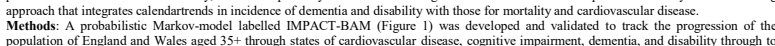

population of England and Wales aged $35+$ through states of cardiovascular disease, cognitive impairment, dementia, and disability through

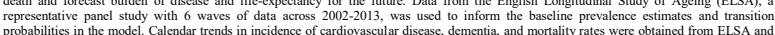

probabilities in the model. Calendar trends in incidence of cardiovascular disease, dementia, and mortality rates were obtained from ELSA and
data from the UK Office for National Statistics and projected to the future. Disability was defined as inbbility to independently perform one

more basic activities of daily living

Results: Average Life-expectancy is estimated at 21.8 year in 2017 and projected to increase to 26.4 years by 2040 (Figure 2 ). The proportion o
disabled to disability-free life expectancy declined over time suggesting compression of morbidity. Using IMPACT-BAM we estimate

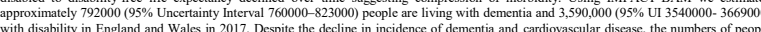

with disability in England and Wales in 2017 . Despite the decline in incidence of dementia and cardiovascular disease, the numbers of peopl
living with dementia and disability are projected to increase to 872000 , 1092000 , and 1205000 for dementia and 3771000,4383000 , an
4902000 for disability in 2020 , 2030, and 2040 respectively. Population ageing is a main contributor as the age-standardized prevalence

declining. Conclusion: Despite the decline in age-specific incidence of dementia and cardiovascular disease, the number of people living with dementi and disabilitit in England and Wat
driven by increasing lifeexpectan $y$.

Our projections have important implications for planning to meet future health and social care needs.

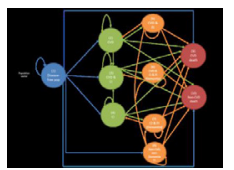

II!I!!

Figure 1: IMPACT-Better Ageing Model (IMPACT-
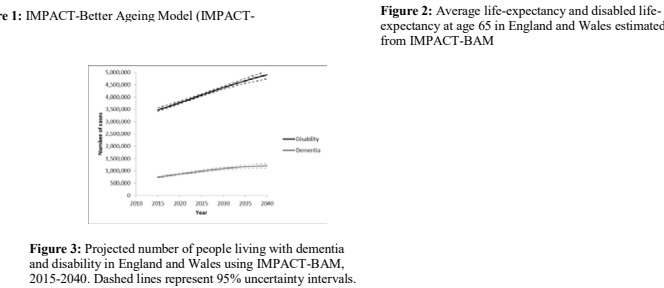

xpectancy at age 65 in England and Wales estimated

\section{Cardiovascular epidemiology of aging}

Pierre Boutouyrie *1

${ }_{1}$ Pharmacologie, Cardiologie - Hôpital Européen Georges Pompidou, Inserm U970, Equipe 7, Paris * Intervenant

This presentation will concentrate on the arterial consequences of arterial aging, especially arterial stiffness, arterial remodeling and wave reflections. We will present the pathophysiological aspects, the different techniques available and the principal results of epidemiological surveys. We will then briefly present some directions for treating early vascular aging.

\section{Neurovascular genetic epidemiology}

\author{
Stéphanie Debette *1 \\ 1 Inserm U1219, Bordeaux \\ * Intervenant
}

With increasing longevity, cerebrovascular disease has become the most common age-related source of disability and dependence, as well as a major determinant of dementia, representing a huge societal burden. Compared to cardiovascular disease, unraveling the genomic underpinnings of cerebrovascular disease has proven more complex due to its much more heterogeneous nature. Recent multiethnic collaborative efforts have enabled important progress in revealing genetic determinants of stroke and MRI-defined covert vascular brain injury. These findings are primarily based on common variants but preliminary results on rare and low-frequency variants are beginning to emerge. Both the "lumping approach" combining all types of strokes with large numbers and strategies focusing on highly specific subtypes of cerebrovascular disease has proven efficient, with complementary findings. Analysis of shared genetic variation with other vascular phenotypes has also provided interesting mechanistic insight. Experimentally follow-up of some of these findings is generating novel hypotheses on the biological pathways underlying stroke. 


\title{
Vascular and thrombosis genetic epidemiology
}

\author{
David-Alexandre TREGOUET $* 1$ \\ 1 Inserm UMR S 1166, Paris \\ * Intervenant
}

During the last 30 years, the evolution of the genetic epidemiology discipline paralleled the development of high-throughput technologies. In the 80 's, familial linkage analysis and candidate gene association approaches were the method of choice for identifying genetic markers associated to human complex diseases. In the last 10 years, high density arrays, followed now by next generation sequencing techniques, allowed the screening for (epi-)genetic and transcriptomic marks that influence disease susceptibility. Henceforth, it is time to interrogate other kind of molecular phenotypes, incl metabolites, proteins, through new high-throughput techniques, and to integrate their analysis with

that of (epi-)genetic data in order to disentangle in a more comprehensive way the complex architecture underlying common human diseases.

This opens the era of the genomic epidemiology discipline or what could be referred to as epidemiolomics.

\section{EVENING PHILOSOPHICAL CONFERENCE ANTONIOMARIO TAMBURRO}

\section{When does the vascular system age and when is there a disease? Conceptual and theoretical issues}

\author{
Maël LEMOINE *1 \\ University of Tours - Philosophy of biological and medical sciences

$$
\text { * Intervenant }
$$

\begin{abstract}
This talk takes a very general perspective in order to question the distinction between aging and disease, in the specific case of the endothelial dysfunction. To what extent is it possible to hold the claim that the difference does not matter insofar as we treat everything we can all the same, whether it is aging or a disease? I will show that it is difficult, but possible to hold it in the face of commonly held but unscientific claims such as "atherosclerosis is aging rather than a disease because it eventually strikes everyone". Then, I will examine the hypothesis that a difference can be made locally between what resorts to aging of the arteries and what resorts to a disease of the arteries, and whether it can

matter. Eventually, I will assess the interest of general biological theories of aging to justify the distinction. In the conclusion, I will try to gather an answer to the question: does it matter to have a distinction between aging and disease of the arteries?
\end{abstract}

\section{PATHOPHYSIOLOGIC FEATURES OF VASCULAR AGING}

\author{
Physiological models to study the human microcirculation - effects of \\ hyperoxya \\ Luis Monteiro-Rodrigues ${ }^{* 1}$ \\ ${ }^{1}$ Universidade Lusófona de Humanidades e Tecnologias - Lisbon, Lisboa, Portugal \\ * Intervenant
}

\begin{abstract}
Models, animal or human, are crucial to look further into the mechanisms involved in a pathological or in a regulatory process to study vascular function and disease, also considering that currently, Vascular Dysfunction represents the 3rd major cause of cerebral-vascular disease. In the last years we explored various approaches to measure microcirculation in vivo, as near as possible from the normal physiological state, and identified several determinants. More recent physiological models are helping to improve the experimental conditions and contribute to accelerate knowledge in these areas.

Hyperoxia is a well known strategy in medicine, also used as a stressor to text microcirculatory regulation mechanisms. We have explored several models, human as animal using hyperoxia. More recently, focusing the improvement of neovascularization process following ischemia, we combined hyperoxia and the hindlimb ischemia, using C57/BL6 mice. In the present communication we intend to share some of our latest experience, and data, in these areas in order to better understand microcirculation physiology.
\end{abstract}




\title{
Murine models for investigating vascular ageing
}

\author{
Gilles Faury *1 \\ ${ }^{1}$ Laboratory HP2, Université Grenoble Alpes, France \\ * Intervenant
}

In human beings, investigating the normal or pathological ageing processes is a difficult task because of the long time course of all the implicated mechanisms, in the range of decades. The access to the elderly is also uneasy because of the laws regulating investigations in patients. In order to overcome these difficulties, numerous murine models have been designed or obtained, by genetic engineering or natural/classical selection. Regarding the investigations of normal ageing, some models allow for easier longitudinal studies because of a selected shorter lifespan, such as senescence-accelerated mice (SAM mice), whereas other models aim at the characterization of the involvement of specific target/candidate mechanisms by gene modification of invalidation, such as Klotho-/- mice. Regarding pathological ageing, or age-related pathologies, a series of models are used, mostly aiming at targeting specific actors (by invalidation, over-expression, ...), such as the mice knockout for the Apolipoprotein E (ApoE-/-, for studies on atherosclerosis) mice or Spontaneously Hypertensive Rats rats (SHR, for studies of the mechanisms or consequences of hypertension). Some other murine models, not overtly related to the ageing, have also brought some insight into the possible modulations of or alternatives to the normal ageing processes, such as the mice heterozygous for the elastin gene (Eln+/-). However, most of these models present some undesirable side-effects which could lead to biases in the possibilities to generalize to the ageing processes that are present in "normal" animals.

The presentation will review some of the main uses of and results from several murine models widely used in the studies of (cardio-) vascular ageing.

\section{New insights into vascular biology by single cell RNA Sequencing}

\author{
Michael VANLANDEWIJCK ${ }^{* 1}$ \\ ${ }^{1}$ Department of Immunology, Genetics and Pathology, Vascular Biology; Research group Christer Betsholtz, \\ Uppsala University, Sweden \\ * Intervenant
}

\begin{abstract}
Cerebrovascular disease is the third most common cause of death in developed countries, yet our understanding of the cells that make the cerebral vasculature is limited. Using large-scale analysis of vascular single cell transcriptomes, we provide molecular definitions to the principal blood vascular and vessel-associated cell types in the adult mouse brain. We uncover the transcriptional basis for the gradual phenotypic change (zonation) that occur along the arterio-venous (A-V) axis in the two major vascular cell types - endothelial and mural cells and reveal unexpected differences between them: a seamless endothelial continuum, as opposed to a punctuated mural continuum. The latter reflects two distinct mural cell groups comprising arterial/arteriolar smooth muscle cells, and pericytes/venous smooth muscle cells, respectively. Brain pericytes are highly homogenous, but a comparison between brain and lung reveals significant pericyte organotypicity. For example, brain pericytes express a unique repertoire of molecular transporters, suggesting their active role at the blood-brain barrier. We also define a population of perivascular fibroblast-like cells that form a previously unrecognized adventitial layer in the brain vasculature. These cells are distinct from mural cells, occur on all vessel types except capillaries and provide distinct anatomical landmarks at the arteriolar-capillary and capillary-venular borders.
\end{abstract}

\section{Summary Thrombosis in Aging}

\author{
Chloé James ${ }^{* 1}$ \\ Inserm U1034, Biology of Cardiovascular Diseases, Pessac - Bordeaux \\ * Intervenant
}

Age is a well-known risk factor for thrombosis, both in the arterial (acute myocardial infarction, stroke) and the venous (deep vein thrombosis, pulmonary embolism) system. Because ageing in humans in most cases occurs in parallel to other thrombotic risk factors (such as hypertension, obesity, cancer, diabetes), the "true" effects of ageing per se may not be easily dissected.

Recently, epidemiologic studies have suggested that aging "per se" is a thrombotic risk factor.

Based on the literature, we will expose how ageing can favour thrombosis, and especially focus on the role of the endothelium, platelets, and coagulation.

\section{MATRIX STRUCTURAL AND MECHANICAL STUDIES}

\section{Model of pseudoxanthoma elasticum}

\author{
Ludovic Martin ${ }^{* 1}$ \\ ${ }^{1}$ CHU Angers \\ ${ }^{*}$ Intervenant
}

Pseudoxanthoma elasticum (PXE) is a prototype of heritable ectopic mineralization. This rare, monogenic (autosomal recessive) condition presents with intriguing manifestations in the skin, retina and cardiovascular systems. Notably, all these systems are rich in elastic tissue and PXE pathological changes are closely related to elastic fibers. The causative gene of PXE is ABCC6, which encodes an ATP-binding transporter primarily expressed in the liver. PXE looks a metabolic condition with central defect in the liver and remote mineralization in the above mentioned systems. The reality might be more complicated... This talk will briefly overview the clinical presentation of PXE, review the specific pathomechanisms operative in ectopic mineralization, including the central role of inorganic pyrophosphate, and discuss whether or not PXE might be seen as an example of premature aging. 


\title{
Vascular calcification and aging, from gene to bedside
}

\author{
Georges Leftheriotis *1 \\ 1 CHU Angers \\ * Intervenant
}

Arterial calcification is a widely demonstrated independent risk factor associated to the cardiovascular morbi-mortality. Calcification of the intimal or medial (i.e. so called mediacalcosis) layers of the arterial wall are frequently observed in acquired metabolic diseases such as chronic kidney disease or diabetes.

Since the last decade, our knowledge of the biological mechanisms underlying this degenerative process has considerably widened with the study of several rare inherited calcifying diseases. For example, the study of PXE, generalized calcification in infancy (GACI) or Arterial Calcification due to Deficiency of CD73 (ACDC) have pointed the importance of the ectonucleotidic regulation of the calcifying process. The complex role of the cell transdifferentiation has been recently evidenced in inflammatory conditions whereas atrogenic induction or protection of calcification are still under the scope of epidemiological studies. Our lecture will focus on the different facets and current concepts of the underlying complex biomineralizing mechanisms and their therapeutic perspectives.

Recommanded readings before the lecture are accessible here:

http://jasn.asnjournals.org/content/15/12/2959.full.pdf

http://ac.els-cdn.com/S147148921630008X/1-s2.0-S147148921630008X-main.pdf? tid=5de16b18-

e692-11e5-9c9f-00000aab0f01\&acdnat=1457595382_5827a2f8e420f132701fcee7fc6a31b7

\section{Vascular aging: structure and dynamics of constitutive macromolecules and sub-fragments}

\author{
V. Samouillan*1, J. Dandurand1, C. Lacabanne1, M. Spina2, V. Llorente-Cortes3, B. \\ Bochicchio $^{4}$, A. Pepe $^{4}$ \\ ${ }^{1}$ CIRIMAT, Université Paul Sabatier, Physique des Polymères, Toulouse \\ ${ }^{2}$ Dipartimento di Scienze Biomediche Sperimentali, Università di Padova, Viale Colombo, Padova, Italy. \\ Institute of Biomedical Research of Barcelona - Spanish National Research Council, Barcelona, Spain. \\ ${ }^{4}$ Dipartimento di Scienze, Universita degli Studi della Basi licata,Potenza, Italy
}

The main constitutive macromololecules of the cardiovascular tissues, essential to the cohesion and resiliency, are also active components which can evolve with ageing.

In the first part of this talk we will show how vibrational, calorimetric and dielectric techniques can be adapted to characterize elastin and In the first part of this talk we will show how vibrational, calorimetric and dielectric techniques can be adapted to characterize elastin and collagens. In peculiar we will see that the vibrational spectra of elastin and type I collagen allow to discriminate between their secondary
structures. Using Differential Scanning Calorimetry (DSC), the thermal fingerprints of elastin and collagens evidence the strong correlation between chain architecture and mesophase organization (Fig 1).

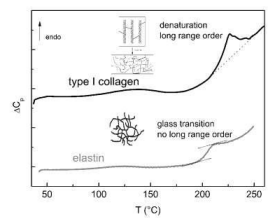

Figure 1: DSC thermograms of freeze dried collagen and elastin

Dielectric techniques reveal the distinct dynamics of these proteins, correlated to their hydrogen bonds network1. This set of data collected on pure proteins is used to help the understanding of vascular aging trough different examples.

One application deals with the use of DSC to compare the thermal signature of elastin and elastin fractions from human thoracic aortas during aging. It is shown that in the thoracic aortas from children group, proteins induce a more flexible structure in contrast with that observed in the thoracic aortas from adults group, due to the total cross-linking of elastin. In this case, there is a segregation between proteins and elastin. No significant differences can be found between the glass transition temperature of elastin in adults and old persons groups, meaning that the in sheet or helical structured proteins is detected with aging, and corroborated by biochemical analyses which report an important increase of polar amino acids in the elastin-proteins fraction of elderly patients2. In this case the protein fraction would form an homogenous phase with elastin by ionic bonds. A second application deals with the combined use of FTIR, DSC and dielectric techniques to characterize a sub fragment of elastin involved in pathological aging. Under certain pathological conditions elastin is attacked by the metalloproteinase MMP12 releasing short polypeptides able to give rise to amyloid-like fibers. Amyloid fibrils are highly ordered parallel cross $\square$-sheet structures usually associated with Alzheimer's disease, Parkinson's disease, type II diabetes. Peculiar signatures of the amyloid fibers can be detected both at the localized and delocalized levels in the condensed state: DSC analysis evidences that S4 peptides are amorphous whereas S4 fibers present a long range order (Fig. 2). Their self-assembly into amyloid fibers lead to macroscopic dipole moments explaining the enhancement of these dipolar properties3 (Fig.3).

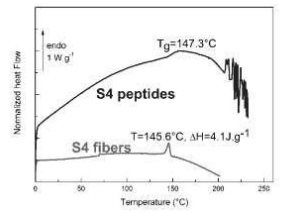

Figure 2: DSC thermograms of S4 peptides and fibers

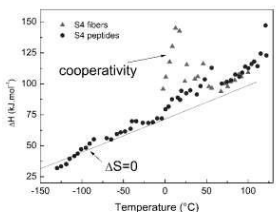

Figure 3: Fine structure analysis of S4 peptidesand fibers

In the last part, we will show how the dielectric techniques, coupled with thermal and vibrational analyses, allow to characterize vascular tissues themselves, both at the nanometric and mesoscopic levels, in the physiologic and pathologic states; this is promising to track vascular aging and also to optimize the conception of substitutive biomaterials.

References

1. Samouillan V, Lamure A., Lacabanne C. Dielectric relaxations of collagen and elastin in the dehydrated state. Chem Phys 2000;255:259-271.

2. Samouillan V, Dandurand J, Lacabanne C, Stella A, Gargiulo M, Degani A, Gandaglia A, Spina M. Analysis of the molecular mobility of

collagen and elastin in safe, atheromatous and aneurysmal aortas. Pathol Biol (Paris) Elsevier Masson SAS; 2012;60:58-65.

3. Samouillan V, Dandurand J, Causse N, Lacabanne C, Bochicchio B, Pepe A. Influence of the Architecture on the Molecular Mobility of

Synthetic Fragments Inspired from Human Tropoelastin. IEEE Trans Dielectr Electr Insul 2015;22:1427-1433. 


\title{
Tubular scaffolds for applications of vascular tissue Engineering
}

\author{
Vicente La Carrubba *1 \\ ${ }^{1}$ DICAM, Università di Palermo, Viale delle Scienze building 8, 90128 Palermo \\ * Intervenant
}

Polymeric tubular scaffolds have been prepared through phase-separation based methods, namely Diffusion Induced Phase Separation (DIPS) and particulate leaching.

According to the DIPS protocol the scaffold, which present a vessel-like shape, can be obtained obtained by performing a phase separation process (induced by a decrease of solvent power) around a nylon fibre covered by a viscous polymeric solution. The fibre was first immersed in a PLLA/dioxane solution (dipcoating bath) at a fixed temperature. Then the fibre was slowly drawn at a constant rate from the solution and put in a second bath (DIPS bath), containing distilled water or dioxane/water at different ratios, at the same temperature, for a well-defined time interval This procedure was repeated once or twice, obtaining scaffolds characterized by a single or double layer respectively, with diameters of 2 and $4 \mathrm{~mm}$.

The scaffolds with double layer are more suitable for vascular tissue engineering applications, due to the more elevated and uniform porosity and the larger mechanical strength.

According to the particulate leaching protocol, the scaffold can be obtained by immersing a fibre in a PLLA/dioxane solution containing NaCl sieved particles with a well defined size, slowly drawing the fibre at a constant rate from the solution and immersing in a second bath (DIPS bath). After drying for 24 hours, the salt particles are extracted via leaching by using sistilled water.

Also in this case single and double layer scaffolds have been prepared with diameters of 2 and 4 mm at various extraction rates. Scaffolds obtained at the extraction rate of $5 \mathrm{~cm} / \mathrm{min}$ via salt leaching are more uniform with respect to the scaffolds produced via DIPS.

Cell cultures (RPMI 2650, nasal mucosa) on scaffolds produced via particulate leaching exhibited better results than the scaffolds obtained via DIPS, with a satisfactory level of adhesion and proliferation and the development of an architecture comparable to the original texture of nasal mucosa in vivo, also confirming the non-cytotoxicity of the used materials.

These preliminary results may address the use of particulate leaching towards vascular tissue engineering applications.

\section{Bioprosthetic heart valve aging deterioration and agerelated Benefits}

\author{
Michel Spina * \\ ${ }^{1}$ Department of Biomedical Sciences, University of Padova, Italy \\ * Intervenant
}

Following population aging and due to congenital and acquired diseases the annual number of patients requiring heart valve replacement worldwide has been estimated to triple from approximately 290,000 in 2003 , to over 850,000 by 2050 [1]. Prosthetic heart valves are broadly divided in mechanical heart valves (MHV) and bioprosthetic heart valves (BHV) while $\approx 55 \%$ are MHVs and $\approx 45 \%$ BHVs $[2]$ not taking into account most recent transcatheter heart valves. MHVs are made of non-biological material while BHVs are either of allogenic (syn. homografts) or xenogenic origin. The last ones are treated with glutaraldehyde to prevent immune rejection. Mechanical devices have a superior durability but the recipients need permanent lifelong anticoagulant therapy. In turn biological valves exhibit excellent hemodynamic behaviour with a low risk of thromboembolic complications but show limited longevity following structural valve degeneration (SVD) particularly in patients $<35$ years of age. The definition of SVD has been associated with the intrinsic permanent changes of prosthetic valve leading to degeneration and/or haemodynamic dysfunction that necessitates reoperation [3]. According to an overall evaluation, 49-63\% of all valve replacements with glutaraldehyde-treated BHVs [3] have been reported to be free from SVD 20 years after intervention. Otherwise the risk of structural failure is inversely related to the age of patients: it is higher in individuals $<35$ years of age in which up to $100 \%$ of BHVs fail within 5 years postsurgery whereas after 15 years $60-70 \%$ of older patients ( $>75$ years) are still free of SVD [4]

In the vast majority of cases SVD is associated with leaflet calcium deposits even if in $19-25 \%$ of BHV implants reoperations occur in the absence of leaflet mineralization [5,6] (excluding nonintrinsic abnormalities, thrombosis and infections). Therefore, considering the elevated levels of oxidized amino acids always associated with bioprosthesis dysfunction, a potentially important role has been prospected also for oxidized amino acid formation [7]. Although not fully understood the mechanism of early failure appears to be complex and multifactorial [6]. The process starts by host IgM/IgG interaction ( $\alpha$-Gal epitope) and macrophage deposition followed by collagen breakdown and finally calcification [8]. Otherwise calcification is believed to be driven by the chemical interaction between phospholipids, residual and oxidized aldehyde groups on one side and circulating calcium ions [4]. A procedure for the suppression of both tissue calcium binding sites and oxidation effects has been developed [9], while to avoid immune injury the use of BHV made of $\alpha$-Gal free tissue (Gal knockout pigs) has been reported [10]. Moreover a novel procedure aimed to overcome comprehensively immune injury, calcification and oxidative modifications has also been recently proposed [11]. At the present only one of these procedures has been implemented in a clinical trial [9, 12].

[1] Yacoub MH and Takkenberg JJM. Will Heart Valve Tissue Engineering Change the World? Nat Clin Pract Cardiovasc Med. 2005; 2: 60-61.

[2] Siddiqui RF, Abraham JR, Butany J. Bioprosthetic heart valves: modes of failure. Histopathology 2009; 55: 135-144.

[3] Capodanno D, Petronio AS, Prendergast B, Eltchaninoff H, Vahanian A et al..

Standardized definitions of structural deterioration or valve failure in assessing long-term durability of transcatheter and surgical aortic bioprosthetic valves: a consensus statement from the European Association of Percutaneous Cardiovascular Interventions (EAPCI) endorsed by the European Society of Cardiology (ESC) and the European Association for Cardio-Thoracic Surgery (EACTS). Eur J Cardio-Thoracic Surg 2017; 52: 408-417.

[4] Schoen FJ, Levy RJ. Tissue heart valves: current challenges and future research perspectives. J Biomed Mater Res 1999; 47: 439-465.

[5] Schoen FJ, Kujovich JL, Webb CL, Levy RJ. Chemically determined mineral content of explanted porcine aortic valve bioprostheses: correlation with radiographic assessment of calcification and clinical data. Circulation. 1987;76:1061-1066.

[6] Barbarash O, Rutkovskaya N, Hryachkova O, Gruzdeva O, Ucasova E, et al. Impact of recipient-related factors on structural dysfunction of xenoaortic bioprosthetic heart valves. Pat Pref Adher 2015; 9: 389-399.

[7] Lee S, Levy RJ, Christian AJ, Hazen SL, Frick NE, et al. Calcification and oxidative modifications are associated with progressive bioprosthetic heart valve dysfunction. J Am Heart Ass 2017; 6:e005648. DOI:10.1161/JAHA.117.005648.

[8] Konakci K, Bohle B, Blumer R et al. Alpha-Gal on bioprostheses: xenograft immune response in cardiac surgery. Eur J Clin Invest 2005; 35; $17-23$.

[9] Dove J, Dobler D, Davidson J, Wright G. Capping bioprosthetic tissue to reduce calcification. Patent No US2014/0200659 A1.

[10] McGregor C, Byrne G, Rahmani B, Chisari E, Kyriakopoulou K, Burriesci G. Physical equivalencyof wild type and galactose $\alpha$ 1,3 galactose free porcine pericardium; a new source material for bioproisthetic heart valves. Acta Biomater 2016; 41: $2014-209$.

[11] Naso F, Stefanelli U, Buratto E, Lazzari G, Perora A, Galli C, Gandaglia A. Alpha-Gal inactivated heart valve bioprostheses exhibit an anticalcification propensity similar to knockout tissues. Tissue Eng A 2017. 23: 1181-1195.

[12] Puskas JD, Bavaria JE, Svensson LG, Blackstone EH, Griffith B, Gammie JS et al. The COMMENCE trial: 2-year outcomes with an aortic bioprosthesis with RESILIA tissue. Eur J Cardiothorac Surg 2017; 52: 432-439. 


\title{
Elastin modification during vascular aging and pathophysiological consequences
}

\author{
Laurent DUCA * \\ ${ }^{1}$ UMR CNRS / URCA 7369, Reims \\ * Intervenant
}

\begin{abstract}
Cardiovascular diseases (CVD) are the leading cause of death worldwide and represent a major problem of public health. Over the years, life expectancy has considerably increased throughout the world and the prevalence of CVD is inevitably rising with the growing aging of the population. The normal process of aging is associated with progressive deterioration in structure and function of the vasculature, commonly called vascular aging. At the vascular level, extracellular matrix (ECM) aging leads to molecular alterations of long half-life proteins, such as elastin and collagen, and have critical impacts on vascular diseases. Elastin, is an insoluble and hydrophobic protein mainly produced by smooth muscle cells in the media and fibroblasts in the adventitia. As elastogenesis is restricted to fetal and infancy, mature elastin fibers remain for lifespan. Indeed, its strong reticulation makes elastin a highly stable molecule with longevity comparable with human lifespan and any proteolytic damage that does occur with age and disease is essentially irreparable. Under pathological conditions, vascular and inflammatory cells produces elastases able to degrade elastin, leading to the production of elastin derived peptides (EDPs) that actively participate through the Elastin Receptor Complex (ERC) to the development of diseases such as diabetes, atherosclerosis, thrombosis and vascular calcification.

Consequently, the proactive role of the ECM suggests that the development of a prognostic value and an innovative therapy based on matrix biology has a strong potential. Finally, some modifications affecting elastin such as glycation or carbamylation could occur and possibly affect the elastin network. The works presented here will summarize and discuss these points.
\end{abstract}

\section{VIVA EVENING CONFERENCE}

\section{History and conceptual developments in vascular biology and angiogenesis research}

\author{
Andréas Bikfalvi * \\ ${ }^{1}$ Inserm U1219, University of Bordeaux, Talence \\ $*$ Intervenant
}

\begin{abstract}
Vascular biology is an important scientific domain that has gradually penetrated many medical and scientific fields. Scientists are most often focused on present problems in their daily scientific work and lack awareness regarding the evolution of their domain throughout history and of how philosophical issues are related to their research field. In this article, I provide a personal view with an attempt to conceptualize vascular development research that articulates lessons taken from history, philosophy, biology and medicine. I discuss selected aspects related to the history and the philosophy of sciences that can be extracted from the study of vascular development and how conceptual progress in this research field has been made. I will analyze paradigm shifts, cross-fertilization of different fields, technological advances and its impact on angiogenesis and discuss issues related to evolutionary biology, proximity of different molecular systems and scientific methodologies. Finally, I discuss briefly my views where the field is heading in the future.
\end{abstract}

\section{CELL BIOLOGY AND SIGNALING}

\section{Estrogens as vascular anti-ageing hormones? Experimental and clinical evidences and uncertainties}

\author{
Jean-François Arnal *1 \\ ' Inserm U1048 - CHU Rangueil, Toulouse \\ * Intervenant
}

\begin{abstract}
Aging is associated with structural and functional changes in the vasculature, including endothelial dysfunction, arterial stiffening and remodeling, impaired angiogenesis, and defective vascular repair, and with increased prevalence of atherosclerosis.

Cardiovascular risk is similar for older men and women, but lower in women during their fertile years.

In women, vascular aging seems to be accelerated during the menopause transition, particularly around the late perimenopausal period. This ageand sex related difference points to estrogen as a protective factor because menopause is marked by the loss of endogenous estrogen production. Experimental studies have attributed part of the protective effects of estrogen to its modulatory action on vascular endothelium. Indeed, estrogen promotes endothelial-derived NO production through increased activity of endothelial NO synthase. However, inhibition of endothelial NO production does not really impair the atheroprotective actions of estrogens. In fact, numerous other cell targets can mediate the actions of estrogens, from smooth muscle cells to various immune cells... Modulations of numerous other factors, such as prostacyclin and hromboxaneA2 release, were also reported and could play a role.

In contrat to the previous epidemiological and observational evidences, the only interventional trial (Women Health Initiative) failed to report any cardiovascular benefit from estrogen replacement therapy, and revealed even an increase in CV events in the older postmenopausal women (i.e. women treated 20 years or more after menopause). This raised the "Timing Hypothesis" states that estrogen mediated vascular benefits occur only before the detrimental effects of aging are established in the vasculature, and could offer a possible « explanation» for these discrepancies. Nevertheless, a gap remains in current knowledge of cardiovascular aging mechanisms in women.
\end{abstract}

The talk will attempt to summarize clinical and experimental data on the effects of aging, estrogens, and hormone replacement therapy on vascular function of females. 


\title{
Arteriolar and microcirculation aging
}

\author{
Daniel HENRION *1 \\ ${ }^{1}$ CNRS UMR 6015 - Inserm U1083, University of Angers \\ * Intervenant
}

Resistance arteries are the small blood vessels located upstream from capillaries. They are crucial for the delivery of blood to vital tissues at relevant flow and pressure. Disorders in resistance arteries structure and function raise capillary pressure and cause downstream organ damage such as that seen in diabetes, hypertension or kidney disease, whose frequency increases with age. Resistance arteries have a basal tone allowing a tight control of local blood flow. This basal tone results of the interaction between myogenic tone due to the activation by pressure of vascular smooth muscle contractility and flowmediated dilatation (FMD) due to the activation of endothelial cells by shear stress. A reduced FMD is the hallmark of endothelium dysfunction. FMD is altered in ageing and in both cardio-vascular and metabolic disorders. Long-term decreases or increases in blood flow induce inward or outward remodelling of resistance arteries respectively. Although flow-mediated outward remodelling is essential in post-ischemic revascularization, it is altered in ageing and this reduced responsiveness is aggravated by the main risk factors. These latter increase oxidative stress and inflammation at the tissue level leading to alter FMD and then to inward remodelling which in turn accelerates oxidative stress and inflammation. Breaking this vicious circle is a major challenge in most countries with a rise in longevity and a high level of exposure to the risk factor associated to the so-called Western way of life. Recent studies have identified the estrogens receptors has key modulators of both FMD and flow-mediated outward remodelling independently of their effect on reproduction, thus opening a new field of investigation in the search for more accurate therapeutic tools reducing vascular ageing.

\section{Oxidation and Inflammation in Atherosclerosis}

\author{
Antonio Junior Lepedda *1 \\ 'Department of Biomedical Sciences - University of Sassari, Sassari, Italy \\ * Intervenant
}

\begin{abstract}
Atherosclerosis is a chronic inflammatory condition, characterized by the accumulation of lipids and fibrous elements in the intimal layer of medium and large arteries, that could turn into an acute clinical event due to plaque ulceration and thrombosis. Indeed, plaque rupture is the predominant underlying process in the pathogenesis of acute coronary syndromes and peripheral vascular disease. Although a great deal of work has been done on it, the mechanisms underlying plaque formation and progression to advanced lesions are not yet completely known. It is generally held that plaque instability is caused by a substantial increase in inflammatory and proteolytic activities. Furthermore, some lines of evidence suggest that unstable plaques are also characterized by pronounced oxidative environment. In situ oxidative events may determine lipid/protein metabolic fate, bioactivity, and antigenic properties. In this respect, oxidized LDL is readily internalized by macrophages through the so-called "scavenger receptor" pathway. These early modifications could initiate and/or contribute to atherogenesis, mainly when an imbalance between oxidant and antioxidant agents takes place. Although several studies report that atherosclerotic plaques contain high concentrations of some amino acid oxidation products, caused mainly by carbonylation, ROS, and RNS oxidation or thiolation, limited information are available regarding the relationship between the accumulation of markers of oxidized proteins and atherosclerosis severity. Furthermore, different oxidation-specific epitopes can be detected in blood and may reflect atherosclerosis manifestations. At present, the mechanisms underlying the formation of these by-products and the relevance for disease progression are under investigation.

Although oxidative stress has been long associated with the genesis and progression of the atherosclerotic plaque, scanty data on its in situ effects on protein sulfhydryl groups oxidation are available. In this respect, reduced protein sulfhydryls, which are in most cases key functional groups, represent a target for several post-translational oxidative modifications. In the last years, we investigated the entity of these oxidations, focusing on low molecular weight thiols adduction, in advanced human carotid plaques, also in relation with plaque vulnerability. By this way, we evidenced deep differences between stable and unstable atherosclerotic plaques as well as between extractable plaque proteins and plasma proteins. Furthermore, by analysing both circulating and plaque-filtered serum albumin (HSA), we evidenced that the pro-oxidant environment present in atherosclerotic tissue could modify filtered proteins also by protein-SH group oxidation, probably contributing to plaque progression. In this regard, our studies suggest, for the first time, that once filtered, albumin represents a harmful source of homocysteine and cysteinylglycine inside the plaque environment. In this respect, the contribution of GSH to the intra-plaque protein-bound LMW thiols equilibrium seems to be particularly relevant.
\end{abstract}




\title{
SELECTED ORAL PRESENTATION
}

\section{MITOCHONDRIAL FUNCTION REGULATES VASCULAR AGING IN MICE}

\author{
Kirsty Foote*1, Johannes Reinhold ${ }^{1}$, Emma Yu ${ }^{1}$, Nichola Figg ${ }^{1}$, Alison Finigan ${ }^{1}$, Mike \\ Murphy $^{2}$, and Martin Bennett ${ }^{1}$ \\ ${ }^{1}$ Department of Cardiovascular Medicine, University of Cambridge - Addenbrooke's Centre for Clinical \\ Investigation Addenbrooke's Hospital Hills Road Cambridge CB2 0QQ, Royaume-Uni \\ ${ }^{2}$ MRC Mitochondrial Biology Unit, University of Cambridge - Addenbrooke's Hospital Hills Road CB2
}

0QQ, Royaume-Uni

\begin{abstract}
Résumé
Mitochondrial DNA (mtDNA) damage is present in aging tissues and may promote loss of tissue function. However whether mtDNA damage or mitochondrial dysfunction is present in the aging vasculature or contributes to vascular aging is unknown. We aimed to determine the time course of functional and structural changes of normal vascular aging in mice, the effect of vessel aging on mitochondrial function, and whether decreased or increased mtDNA damage delays or promotes vascular aging respectively. Wild-type (WT) C57Bl/6 mice were studied at $8,22,44$ and 72 wk of age by ultrasound imaging and intra-arterial blood pressure measurements using single and dual pressure catheters to provide a range of functional parameters of vascular aging. Vascular aging was detected in WT mice between $22-44 \mathrm{wk}$ of age, with reduced carotid arterial compliance and distensibility and increased b stiffness index (bSI), and increased aortic pulse wave velocity (PWV) (all $P<0.05$ ). No additional changes were noted between 44-72wk. Aortic collagen content and elastin breaks also increased by $57 \%$ and 4 -fold respectively (both $P<0.05$ ) between $22-44$ wk. Similarly, mtDNA copy number assessed by quantitative PCR decreased significantly between $22-44 \mathrm{wk}$ $(P<0.05)$ and mitochondrial respiration assessed by a Seahorse flux analyser decreased by $25 \%(P<0.05)$. To determine the effects of mtDNA damage, we studied vascular aging in mice that overexpressed the mitochondrial helicase Twinkle (Tw+), or with a mutation in the proof-reading ability of the mitochondrial polymerase gamma (PolG). Twinkle overexpression restored mtDNA copy number with concurrent improvement in mitochondrial respiration, and delayed all physiological parameters of vascular aging associated with decreased collagen and elastin breaks $(P<0.05)$. In contrast, PolG mice with increased mtDNA damage showed accelerated vascular aging compared to controls $(P<0.05)$. We have identified multiple, reproducible parameters of arterial aging in mice that are detected at far earlier time points than previously described; in particular, compliance, distensibility and bSI at 44wk provide the earliest discrimination. Arterial mitochondrial function reduces markedly with age, and accelerates vascular aging, whereas augmenting mitochondrial function delays aging, identifying prevention of mtDNA damage and dysfunction as a therapeutic target in aging.
\end{abstract}

Mots-Clés: vascular, aging, mitochondria

*Intervenant. 


\section{Structural imaging of the vascular wall}

Azzam Alwann', Amandine Wahart ${ }^{2}$, Sébastien Blaise ${ }^{3}$, Edith Grall-Maës ${ }^{4}$, Béatrice Romier $^{5}$, Pierre Beauseroy ${ }^{4}$, Laurent Duca ${ }^{5}$, Michael Sheratt ${ }^{6}$, Laurent Debelle ${ }^{5}$, Manuel Dauchez ${ }^{7,8}$, Rémi Cogranne $^{9}$ and Sébastien Almagro* ${ }^{* 10}$

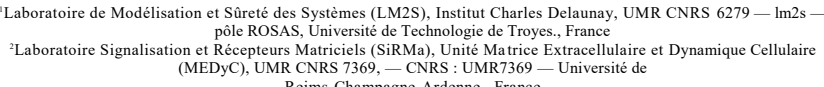

\section{Numerical assessment and comparison of pulse wave velocity methods aiming at measuring aortic stiffness}

Résumé

Numerical assessment and comparison of pulse wave velocity methods aiming at measuring aortic stiffness

Pulse waveform analyses have become established components of cardiovascular research Recently several methods have been proposed as tools to measure aortic pulse wave velocity (aPWV). The carotid-femoral pulse wave velocity (cf-PWV), the current clinical gold standard method for the noninvasive assessment of aPWV, uses the carotid - to - femoral pulse transit time difference (cf-PTT) and an estimated path length to derive cf-PWV. The heart-ankle PWV (ha-PWV), brachial-ankle PWV (ba-PWV) and finger-toe (ft-PWV) are also methods presuming to approximate aPWV based on time delays between physiological cardiovascular signals at two locations (_ heart-ankle PTT, ha-PTT; brachial-ankle PTT, ba-PTT; - finger-toe PTT, ft-PTT) and a path length typically derived from the subject's height. To test the validity of these methods, we used a detailed 1D arterial network model (143 arterial segments) including the foot and hand circulation. The arterial tree dimensions and properties were taken from the literature and completed with data from patient scans. We calculated PTT's with all the methods mentioned above. The calculated PTT's were compared with the aortic PTT (aPTT), which is considered as the absolute reference method in this study. The correlation between methods and aPTT was good and significant, cf-PTT $\left(\mathrm{R}^{2}=0.97 ; \mathrm{P}<0.001\right.$; mean difference $\left.5+2 \mathrm{~ms}\right)$, ha-PTT $\left(\mathrm{R}^{2}=0.96 ; \mathrm{P}<0.001 ; 150+23\right.$ $\mathrm{ms})$, ba-PTT $\left(\mathrm{R}^{2}=0.96 ; \mathrm{P}<0.001 ; 70 \pm 13 \mathrm{~ms}\right)$ and ft-PTT $\left(\mathrm{R}^{2}=0.95 ; \mathrm{P}<0.001 ; 14 \pm\right.$ $10 \mathrm{~ms}$ ). Consequently, good correlation was also observed for the PWV values derived with the tested methods, but absolute values differed because of different path lengths used. In conclusion, our computer model based analyses demonstrate that for PWV methods based on peripheral signals, pulse transit time differences closely correlate with the aortic transit time, supporting the use of these methods in clinical practice. 


\title{
Long-term trajectories of cardiometabolic risk factors in prodromal dementia: the Three-City Study
}

\author{
Maude Wagner ${ }^{1,2}$, Catherine Helmer ${ }^{2}$, Christophe Tzourio ${ }^{3}$, Claudine Berr ${ }^{4}$, Cécile \\ Proust-Lima $^{1^{*}}$, and Cécilia Samieri ${ }^{2 *}$ \\ ${ }^{1}$ Univ. Bordeaux, Inserm U1219, Bordeaux Population Health Research Center team Biostatistics \\ ${ }^{2}$ Univ. Bordeaux, Inserm U1219, Bordeaux Population Health Research Center team LEHA \\ ${ }^{3}$ Univ. Bordeaux, Inserm U1219, Bordeaux Population Health Research Center, Team HEALTHY \\ ${ }^{4}$ Univ. Montpellier, Inserm U1061, Neuropsychiatry: Epidemiological and Clinical Research \\ *These authors contributed equally to this work
}

\begin{abstract}
Objective: To describe the trajectories of major cardiometabolic risk factors (body mass index [BMI], systolic and diastolic blood pressure [SBP, DBP], fasting blood lipids [HDL and LDL cholesterol, triglycerides] and glycemia) in the 14 years preceding dementia diagnosis, and to compare them to the trajectories of matched subjects free of dementia, using latent process mixed models.

Design: Case-control study nested in a prospective cohort.

Setting: The Three-City Study, a population-based cohort of older persons ( $\geq 65$ years) enrolled between 1999 and 2001 in three French cities (Bordeaux, Dijon, and Montpellier) who underwent up to six repeated home visits with neuropsychological testing and measurements of anthropometric parameters and blood pressure, and up to three repeated blood draws for assessment of biological parameters.

Participants: 785 incident cases of dementia and with evaluation of cardiometabolic risk factors at baseline individually matched (for gender, age, education, and study center) to 3,140 controls at the time of dementia cases diagnosis.

Results: In both cases and controls, BMI, DBP and HDL declined in the 14 years preceding the matching visit, whereas SBP and glycemia increased and LDL and triglycerides remained stable over time.

Compared to controls, dementia cases had a faster decline in BMI and a lower increase in SBP $(P<.001$ and $P<0.049$ respectively for group-bytime interactions) leading to significantly lower BMI and SBP values among cases respectively 2 and 4 years before diagnosis. The other cardiometabolic risk factors (DBP, lipids and glycemia) did not evolve differently according to disease status (all $P$ for group-bytime

interactions $>0.20$ ); however cases presented lower DBP and higher fasting glycemia levels over the entire period.

Conclusion: Higher BMI, blood pressure and blood lipids were not found as strong risk factors for dementia in the older age range; on the contrary BMI and blood pressure decreased months to several years before dementia diagnosis (possibly as a consequence of changes in the prodromal phase of the disease). Blood glucose was the single cardiometabolic risk factor with constantly higher values over the 14 years before diagnosis among incident dementia cases, suggesting that elevated glycemia is among the strongest cardiometabolic risk factor for dementia in older persons.
\end{abstract}

\section{MicroRNA expressed in progenitor cells circulating in peripheral blood as prospective biomarkers of agerelated macular degeneration (AMD) in its dry and wet form - preliminary data}

\author{
A. Machalińska1, M.P. Kawa*2, A. Sobuś2, Z. Litwińska2, A. Kowalska-Budek1, K. Babiak2, \\ A. Grabowicz2, B. Machaliński2 \\ 1. First Department of Ophthalmology, \\ 2. Department of General Pathology, \\ Pomeranian Medical University, Szczecin, Poland \\ *Intervenant
}

\begin{abstract}
:
Objectives: Age-related macular degeneration (AMD), a worldwide public health problem, has been recognized as a common cause of visual dysfunction in elderly people. MicroRNAs (miRNAs) present in sera are characteristically altered in many pathological conditions and have been used as biomarkers for specific diseases, including AMD. Several miRNAs can be detected in bodily fluids including serum, urine and saliva, where they are released in form of exosome vesicles. However, different physical conditions, including blood oxygen tension, alter the exosome release and affect the global miRNA profile in fluids modifying their bioactivity as a consequence. In contrast, being resistant to exonucleolytic activities, intracellular miRNAs are more stable and have longer half-life than extracellular miRNA, therefore may play a role in development and progression of diseases. Due to the high abundance, stable and accessible characteristics, intracellular miRNAs could serve as putative biomarker molecule in clinically relevant samples. Thus, in our study, we analyzed selected miRNAs from peripheral blood mononuclear cells (PBMCs) in patients with dry and wet form of AMD and healthy volunteers (HV), and we investigated the intracellular miRNAs expression as potential biomarker for early diagnosis of dry or wet form of AMD.

Material and Methods: PBMCs were collected from 94 AMD patients ( 46 wet form and 48 dry form) and $46 \mathrm{HV}$ as a source of miRNAs. Selfvalidated individual quantitative RT-PCR study was then performed to evaluate the expression levels of selected miRNAs. The Mann-Whitney $\mathrm{U}$ test was used for statistical analysis.

Results: miRNA expression analysis revealed a decreased expression of selected angiogenesis-related miRNAs in both forms of AMD, including miR-223-3p, miR-17-5p (both greatly down-regulated in dry AMD), and miR-150-5p (stronger down-regulation in wet AMD). In contrast, two angiogenic miRNAs: miR126-5p and miR126-3p were upregulated, especially in dry AMD.

Conclusion: Our results indicated that altered expression of selected miRNAs may play a role in the AMD development and progression. Further studies are required to determine causal relationships and elucidate underlying mechanisms. This work was supported by grants: STRATEGMED1/234261/2NCBR/2014 and UMO-2013/09/B/NZ7/04031

Keywords: MicroRNA, age-related macular degeneration (AMD), dry AMD, wet AMD, angiogenesis, intracellular miRNAs, disease biomarkers
\end{abstract}




\section{Molecular, conformational and thermal \\ characterization of ventricular Remodeling in a pig model of tachycardia-induced cardiomyopathy}

Valerie Samouillan', Aleyda Benitez-Amarot", Esther Jorge", Jany Dandurand ${ }^{1}$, Laura Nasarre ${ }^{3}$, David De Gonzalo-Calvo ${ }^{3}$, Olga Bornachea ${ }^{2,3}$, Colette Lacabanne ${ }^{1}$, Jose M Guerra', and Vicenta Llorente-CortesI"

Centre interuniversitaire de recherche et dingenierie des materiaux (CIRIMAT) — Institut National Polytechnique [Toulouse], Universite Paul Sabatier - Toulouse 3, Centre National de la Recherche Scientifique : UMR5085 - Laboratoire de Physique des Poly 118,route de Narbonne 31062 TOULOUSE CEDEX 4, France

${ }^{2}$ Institute of Biomedical Research of Barcelona-Spanish National Research Council (IIBB-CSIC) Rosse116, 1616 ' i $7^{\text {a }}$ planta, 08036 Barcelona, Espagne

${ }^{3}$ Biomedical Research Institute Sant Pau (IIB Sant Pau) - Hospital de la Santa Creu i Sant Pau. San Antoni Ma Claret, 167 Pavello ${ }^{\circ}$ 16, Sant Frederic, 08025 Barcelona, Espagne ${ }^{4}$ Universitat Autonoma de Barcelona (UAB) - Placa Mica, s/n, 08193 Bellaterra, Barcelona, Espagne

*Auteur correspondant: valerie.sarnouillan@univ-tlse3.fi t Intervenant

tAuteur correspondant: cllorente@,csic-iccc.org

Resume

Aims: Non-ischemic dilated cardiomyopathy (NIDCM) is characterized by ventricular dilatation associated to pathological remodeling. Our aim was to compare molecular, conformational and physical characteristics of healthy and NIDCM remodelar

mocardich Methods and Results. Our study includes healthy pigs (control, $N=7$ ) and tachycardia-induced NIDCM pigs (DCM, $\mathrm{N}=10$ ). Samples in collagen $I$ and collagen $\mathrm{HI}$ in both RV (2.06 and 1.38 -fold) and LV (2.43 and 1.48 -fold) dilated ventricles. Fourier Transform Infrared Spectroscopy (FTIR) $1 \mathrm{st}[\mathrm{A}(1171 \mathrm{~cm}-1) / \mathrm{A}(1338 \mathrm{~cm}-1)]$ and $2 \mathrm{nd}[\mathrm{A}(1304 \mathrm{~cm} 1) / \mathrm{A}(1338 \mathrm{~cm}-1)]$ indicators reflect myofiber/collagen ratio while $[\mathrm{A}(1043 \mathrm{~cm}-1) / \mathrm{A}(2800 \mathrm{~cm}-1-3000 \mathrm{~cm}-1)] 3 \mathrm{rd}$ indicator reflect carbohydrate/lipid ratio. 1 st indicator was reduced in both dilated ventricles (to $43 \%$ in RV and to $37 \%$ in LV), while 2nd indicator was only reduced in dilated RV (to $37 \%$ ). 3rd indicator was upregulated in both dilated ventricles, but to a higher extent in RV ( 2.60 -fold vs 1.61 -fold, $\mathrm{P}=0.049$ ). Histological Masson's trichromic staining showed unorganized and agglomerated collagen in dilated ventricles, and a higher percentage of fibrosis in RV compared to LV $(\mathrm{P}=0.016)$. Both FITR and thin layer chromatography revealed the cancellation of the differences in cholesteryl ester and triglyceride content between RV and LV (inherent to control pigs) in DCM group. Differential Scanning Calorimetry (DSC) evidenced a strong decrease of protein denaturation temperature (a shift of $50^{\circ} \mathrm{C}$ in the freeze-dried state) in both dilated ventricles, associated with a significant depression of the freezable water melting point.

Conclusion: Our results point to reduced myofiber/collagen ratio, increased carbohydrate/lipid ratio and lower protein Conclusion: Our restin ventricles. The exacerbation of these alterations in RV underlies deep re dilated cardiomyopathy.

Mots-Clés: Heart failure, cardiac remodeling, myofiber, collagen, FITR, DSC

Valvular calcification: implication of the Semicarbazide-Sensitive Amine Oxidase (SSAO)?

\footnotetext{
Nathalie Mercier ${ }^{* 1}$, John Pirault ${ }^{1}$, Bastien Wollensack ${ }^{1}$, and Magnus Bäck ${ }^{2,3}$

${ }^{1} \mathrm{UMR}_{S} 1116$ IN SERM/universitédeLorraine $(D C A C)-$

-Institut NationaldelaSantéetdela Recherche Médicale - INSERM, Univer sitéde Lorraine -

-FacultédeMédecine, BatD, 1erétage9, avenuedelaForêtde HayeBP5018454505Vandoeuvre - Lès NancyCedex, France

${ }^{2}$ Karolinska University Hospital - Divison of Valvular and Coronary Disease, Karolinska University Hospital, 17176 Stockholm, Sweden, Suède

${ }^{3}$ Karolinska Institutet - Center for Molecular Medicine, Department of Medicine, Karolinska Institutet, 17176 Stockholm, Sweden, Suède
}

Résumé

Introduction: Calcific aortic valve disease (CAVD) leads to aortic stenosis, a common disease associated with aging. CAVD shares similarities with the process that regulates atherosclerosis but the full mechanisms leading to CAVD are still unclear. Apart from surgical replacement of the valve, therapeutic options are limited, calling for new potentia targets involved in calcifications. A relationship between serum Vascular Adhesion Protein (VAP-1) and calcific aortic stenosis has been described but nothing is known on the tissular form of VAP-1. VAP-1, also called "Semicarbazide-Sensitive Amine Oxidase" (SSAO), is an enzyme that transforms primary amine into aldehyde, $\mathrm{H} 2 \mathrm{O} 2$ and ammonia but also functions as an adhesion molecule. Interestingly, VAP-1 has been implicated in inflammatory diseases, atherosclerosis and differentiation process of several cell types. The aim of this study was to highlight the involvement of SSAO in valvular calcifications and to develop a primary culture noolet of intertitint oells of aortic valves (PAVICs) to study the potential molecular

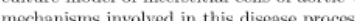

Yethe 13 Ho then

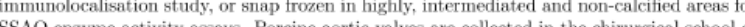
Nancy to establish primary cultures of valvular interstitial cells (VICs).

Results: Our results showed a significant increase in SSAO protein expression and enzyme activity in calcified area of the human aortic valves compared to healthy area of the valve After 14 days of cell culture, SSAO activity is also detectable in PAVIC

Conclusion: This is the first time that tissular SSAO expression and activity has been asso-

ciated with valvular calcification. However, the mechanisms need to be studied in detail.

Mots-Clés: aortic valves, calcifications, aortic valvular intentitial cells, "semicarbazide, sensitive amine oxidase", hydrogen peroxide 
Vascular calcification during chronic kidney disease : role of the RAGE/Cathepsin S/elastin peptides axis

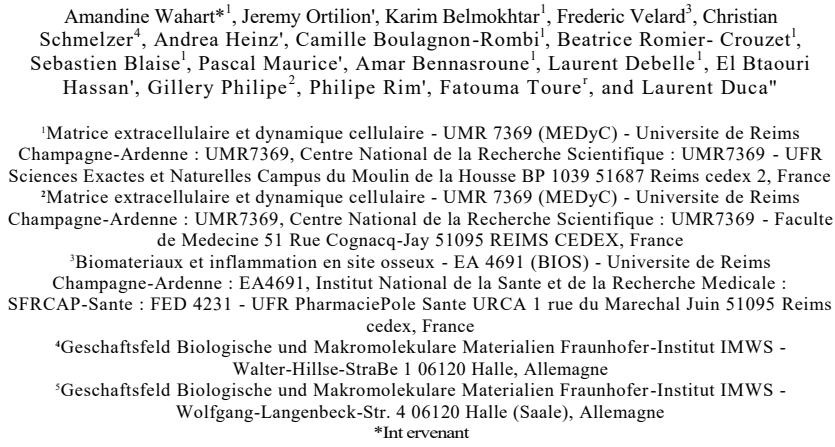

Vascular calcification is a common feature of patients suffering from chronic kidney disease (CKD). We recently reported a role for the Receptor for Advanced Glycation End products (RAGE) in the uremic vascular calcification process. Following engagement by uremic toxins, RAGE mediates active osteodifferentiation of vascular smooth muscle cells (VSMCs) and vascular calcifications. This process is mainly found in aorta where an intense elastolysis is observed leading to the release of elastin derived peptides (EDPs). Using animal models, severa reports from the literature have suggested the possible involvement of Cathepsin $\mathrm{S}$ in elastolysis and vascular calcification during CKD. However, the mechanisms implicated and the putative role of RAGE in Cathepsin S expression and the subsequent elastolysis have not been described.

To answer these questions, we used a mouse model of uremic vasculopathy, induced by 5 of 6 nephrectomy in the ApoE-/- or APoE-/-/RAGE-/- backgrounds. Moreover, we used primary cultures of VSMCs isolated from control or RAGE deleted animals for in vitro studies.

We found that induction of CKD increases the calcification process in the cardiac valves of ApoE-/- mice whereas ApoE-/-/RAGE-/- mice are protected. Moreover, sera and aortas analysis showed that Cathepsin 5 expression and elastolysis seem to be greater in ApoE-/- compared to the ApoE-/-/RAGE-/- animals. Using recombinant Cathepsin $S$, we found that this protease was able to directly drive insoluble elastin degradation and bioactive EDPs production as demonstrated by electron microscopy scanning and mass spectrometry analysis. Finally, we showed that an in vitro calcification process is triggered when VSMCs are incubated with inorganic phosphate. This phenomenon is increased in the presence of EDPs enhancing expression of osteoblast differentiation specific genes.

In conclusion, we report that in uremic conditions, increased Cathepsin S expression may promote elastolysis. This process generates bioactive EDPs which could accelerate osteogenic differentiation of VSMCs and apparition of vascular calcification. RAGE engagement may be involved in the initial steps leading to increased Cathepsin $\mathrm{S}$ production.

Mots-Clés: vascular calcification, RAGE, cathepsin S, elastin derived peptides, chronic kidney disease

\section{Targeting Connexin40 expression or function reduces angiogenesis in the developing mouse retina} Jacques-Antoine Haefliger $^{1}$, Florent Allagnat $^{1}$, Lauriane $_{\text {Hamard }}{ }^{1}$, Elisabeth Génot $^{2}$,
and Florian Alonso

${ }^{1}$ Department of Medicine, Lausanne University Hospital - Lausanne, Suisse ${ }^{2}$ Centre de recherche Cardio-Thoracique de Bordeaux [Bordeaux] (CRCTB) - Université Bordeaux Segalen - Bordeaux 2, CHU Bordeaux [Bordeaux], Institut National de la Santé et de la Recherche Médicale : U1045 - Université Bordeaux Segalen 146 rue Leo Saignat Zone sud, Batiment TP, 2ème étage Case courrier 1333076 Bordeaux cedex, France

Résumé

Introduction: Connexins constitute a large family of trans-membrane proteins that form gap junction channels enabling direct transfer of small signaling molecules from cell-totheir ability to vascularise tumor xenografts. However, how $\mathrm{Cx} 40$ contributes to angiogenesis has not yet been investigated.

Methods: We used the mouse neonatal retina model and Cx40-deficient (Cx40-/-) mice to explore Cx40 expression and function in developmental angiogenesis. We used immortalized mouse brain microvascular ECs to explore the contribution of $\mathrm{Cx} 40$ channels on cell properties and behavior in vitro.

Results: Our data show that $\mathrm{Cx} 40$ is expressed in the immature vascular plexus of the developing retina. Genetic ablation of $\mathrm{Cx} 40$ causes retinal hypovascularization in the postnatal mouse eve, with reduced radial vessel growth and decreased capillary density as comparec to WT mice. At the angiogenic front, Cx40-deficiency attenuates vessel sprouting and the mural cells recruited on the neovessels display an altered phenotype. These alterations can be attributed to disturbed endothelial cell functions as selective re-expression of $\mathrm{Cx} 40$ in these cells restores normal angiogenesis. In vitro, targeting $\mathrm{Cx} 40$ by silencing its expression or by blocking gap junction channels, decreases endothelial cell proliferation, modifie their secretome and promotes mural cell chemoattraction. Finally, a Cx40 inhibitory peptide (40Gap27) phenocopies the loss of endothelial Cx40 in vitro and in vivo.

Conclusions: Our data show that endothelial $\mathrm{Cx} 40$ regulates retinal vessel growth and maturation. $\mathrm{Cx} 40$ thus represents a novel potential target for treating pathological ocular angiogenesis. 


\title{
Imatinib inhibits hypoxia-induced MMP-9 overexpression and activation through impairment of LRP1 and Pyk2 hosphorylation in human coronary vascular smooth muscle cells
}

\author{
Aleyda Benitez-Amare- ${ }^{2}$, Elena Revuelta-Lopez ${ }^{3}$, David De Gonzalo-Calvo ${ }^{2}$, Laura \\ Nasarre $^{2}$, Santiago Roura ${ }^{3}$, Antoni Bayes Genis ${ }^{3}$, and Vicenta Llorente-Cortes"' ${ }^{2}$ \\ Institute of Biomedical Research of Barcelona-Spanish National Research Council (IIBB-CSIC) - \\ Rosse 116, $1616^{\mathrm{a}}$ i 7' planta, 08036 Barcelona, Espagne \\ ${ }^{2}$ Group of Lipids and Cardiovascular Pathology. Biomedical Research Institute Sant Pau (IIB Sant \\ Pau) - Hospital de la Santa Creu i Sant Pau. Sant Antoni Ma Claret, 167 Pave1 16 n $^{\circ}$ 16, Sant Frederic, \\ 08025 Barcelona, Espagne \\ ${ }^{3}$ ICREC Research Group. Institut d'Investigacio en Ciencies de la Salut Germans Trias i Pujol (IGTP) \\ — Cami de les Escoles s/n 08916 Badalona (Barcelona), Espagne \\ *Intervenant \\ tAuteur correspondant: cllorentencsic-iccc.org \\ Resume
}

Introduction. An imbalance in the migration of vascular smooth muscle cells (VSMCs) is associated with the progression of vascular diseases. Low-density lipoprotein receptor-related protein 1 (LRP1) is known to participate in VSMC migration through activation of metalloproteinases (MMPs) and Pyk2 in human VSMCs (hVSMCs). Hypoxia increases hVSMC migration through an upregulatory effect on LRP1-pPyk2MMP-9 axis. Gleevec, a protein kinase inhibitor, reduces the area of the atherosclerotic plaque in diabetic apoE-K0 mice. The aim of this work was to study the effect of Gleevec in LRP1-pPyk2-MMP-9 axis and hVSMC migration.

Methodology. The hVSMCs were maintained in medium 199 supplemented with $20 \%$ fetal bovine serum, exposed to normoxia $(21 \% 02)$ or hypoxia $(1 \% 02)$ and treated with Gleevec $20 \mathrm{p}, \mathrm{M}$ for 20 hours. Protein levels of p-LRP1, LRP1 (b-chain), pPyk2, total Pyk2, pERK1/2, Total ERK1/2 and 3 -tubulin was measured by Western blot. The formation of protein complexes was analyzed by immunoprecipitation experiments. MMP9 and MMP2 expression and activity were tested by Real time PCR and zimography, respectively. Migration studies were performed using the technique of wound repair assays after scratching a cell monolayer

Results. Real time PCR and zimography data showed that hypoxia failed to increase MMP-9 expression and activity in Gleevec-treated hVSMC. Wound repair assays showed that Gleevec prevented the increase on hVSMCs migration induced by hypoxia. Immunoprecipitation and Western blot assays revealed that the amount of LRP1/pPyk2 complexes increased under hypoxic conditions but that hey almost completely absent in Gleevedtreated hVSMC compared to control hVSMC Conclusions. Gleevec effectively prevented hypoxiainduced hVSMC migration. Therefore, Gleevec could be used to counteract the deleterious effects of hypoxia on vascular remodeling.

Mots-Cles: Imatinib, hVSMCs, LRP1, MMP9, Gleevec

\section{NRP1 supresses TGF $\beta$-dependent ERK1/2 activation in endothelial cells}

\author{
Lampropoulou A.1, Raimondi C.2, Fantin A.1, Ruhrberg C $_{1 .}$ \\ ${ }^{1}$ Department of Cell Biology, UCL Institute of Ophthalmology, University College London, 11- \\ 43 Bath Street, London EC1V 9EL \\ ${ }^{2}$ Vascular Sciences Unit, Imperial Centre for Translational \& Experimental Medicine, Imperial College, NHLI, Hammersmith \\ Campus, Du Cane Road, London, W12 ONN
}

\begin{abstract}
Vascular endothelial cells express the transmembrane receptor neuropilin 1 (NRP1) to promote vascular endothelial growth factor VEGF-A and integrin ligand-induced extracellular matrix signalling. Recently, NRP1 was shown to also repress transforming growth factor $\beta$ (TGF $\beta$ ) signalling to modulate endothelial cell signalling. All three pathways cooperatively regulate retinal blood vessel growth. Consistent with prior work, we also find that NRP1 represses TGF $\beta$ signalling. However, in addition to the previously proposed pathway, in which NRP1 loss de-represses canonical SMAD signalling, we find that NRP1 loss also increases endothelial TGF $\beta$ expression and noncanonical TGF $\beta$ signalling via ERK1/2 activation in both mouse and human endothelial cells. Moreover, we show that knockdown of the TGF $\beta$ receptor TGFBR2 or its co-receptor ALK1 blocks NRP1dependent ERK1/2 activation and ERK1/2-dependent gene transcription, a pathway previously shown to accelerate cellular aging. Taken together with prior work, these findings identify NRP1 as a multifunctional regulator of endothelial cell behaviour.
\end{abstract}


The elastin receptor complex interacts with CD36 through NEU1 and regulates oxidized LDL uptake in macrophages: potential implication in the proatherogenic effects of elastin-derived peptides

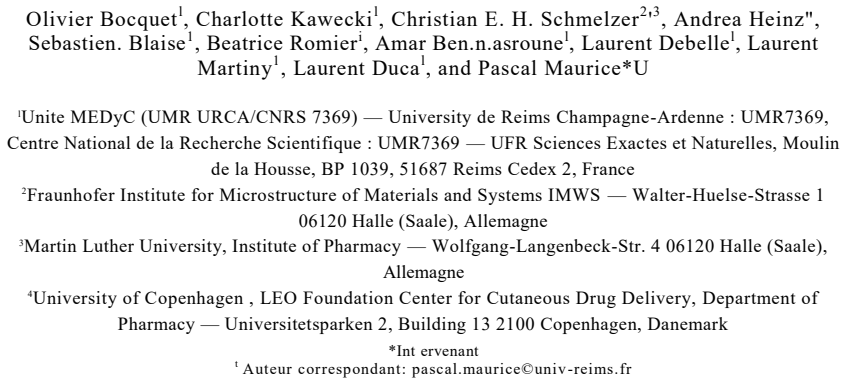

Vascular aging is associated with extracellular matrix (ECM) remodeling and proteolysis of elastin. Elastin is a major ECM component of the arterial wall that provides the elastic recoil properties and resilience essential for proper vascular function. Elastin degradation leads to the generation of elastin-derived peptides (EDP) that exert biological effects through the plasma membrane neuraminidase 1 (NEU1), a sialidase catalyzing the removal of terminal sialic acids from sialyloconjugates. This sialidase interacts with the elastin binding protein and the protective protein/cathepsin A forming the elastin receptor complex (ERC) at the plasma membrane. Results of our lab from the last five years have brought significant insights on the critical role played by NEU1 in signal transduction and pathophysiological effects mediated by the ERC and EDP in vascular diseases such as type 2 diabetes, atherosclerosis and thrombosis.

such as type 2 diabetes, athesosclersis and thrombsis. In this (a) B scavenger receptor CD36 was identified. Interaction between NEU1 and CD36 was confirmed by coimmunoprecipitation and colocalization experiments. Using a lectin-affinity assay, we show that EDP desialylate CD36 and that CD36 desialylation by EDP is blocked by the V14 peptide, a peptide blocking the interaction between bioactive EDP and the ERC. Finally, we show that EDP increase the uptake of oxidized LDL by macrophages that is blocked by both the V14 peptide and DANA, a sialidase inhibitor

In conclusion, these results demonstrate for the first time that the ERC interacts with CD36 through NEU1 at the plasma membrane of macrophages. Binding of EDP to the ERC modulates CD36 sialylation level and regulates oxidized LDL uptake by macrophages through NEU1. These effects could contribute to the previously reported proatherogenic role of EDP in atherosclerosis.

Mots-Cles: elastin peptides, neuraminidase, interaction partners, CD36, macrophages

\title{
UBIAD1 PROMOTES TUMOR ANGIOGENESIS BY INHIBITING DEPHOSPHORYLATION OF VEGFR2 IN A REDOX DEPENDENT MANNER
}

\author{
Raj Nayan Sewduth * \\ ${ }^{1}$ Laboratory of Endothelial Molecular Biology, Belgium \\ * Intervenant
}

Tumor microenvironment accumulate reactive oxidative species (ROS) during tumor formation. This in important for the tumor cells to proliferate and transform but can also challenges the endothelial cells present in the tumor. That's why the tumor endothelial cells need to express antioxidants to compensate for high oxidative level and to limit cellular damage. Tumor endothelial cells are also neoangiogenic as they need to invade surrounding tissues to 'feed' the tumor. This is associated to the activation of the VEGF pathway. We are interested in an antioxidant enzyme called Ubiad1 that is located to the Golgi and important for the biosynthesis of antioxidant molecules Coq9/10 and Vitamin K2. Ubiad1 is mutated in Schnyder crystalline corneal dystrophy (SCCD) where cholesterol accumulates in the cornea leading to blindness. Here we show that Ubiad1 SCCD endothelial cells are more angiogenic leading to formation of bigger tumors when lung carcinoma cells were implanted subcutaneously. This was associated with a higher level of activation of VEGFR2. Antioxidants Coq9/ 10 levels were also higher in Ubiad1 SCCD endothelial cells. High level of antioxidants also induce oxidative modification (glutathionylation) of the phosphatase LMW-PTP, making it inactive. This phosphatase was shown before to dephosphorylate VEGFR2 making it inactive12. Thus we propose a mechanism in which high levels of Ubiad1/ antioxidants in SCCD cells appear to promote activation of VEGFR2 by promoting oxidative modifications of LMW-PTP. This work suggests that manipulation of redox state by targeting antioxidant enzymes provides an innovative approach to block tumor angiogenesis, as inhibition of glutathionylation significantly reduced tumor growth and angiogenesis, in wild-type but not Ubiadl SCCD mice.

Authors: C Millia, X Chen, W Lee, Elisabetta Grillo, Y Jonnson, C Aelbrecht, E Turco, C Medana, MM. Santoro 


\title{
OPEN SESSION
}

\section{Endothelial cell dysfunction is a new potential therapeutic target for the treatment of critical limb ischemia}

\author{
Caroline Caradu *1 \\ 1 Inserm U1034 - Pessac
}

* Intervenant

\begin{abstract}
Therapeutic angiogenesis for ischemic diseases including critical limb ischemia (CLI) failed to demonstrate significant clinical improvement. In the present study, we explored endothelial cell (EC) dysfunction in the pathophysiology of CLI, as a possible alternative therapeutic target. In a systematic histological analysis of CLI human biopsies, we found that CLI muscles were characterized by evident signs of myopathy, but that capillary density within the ischemic muscle was equivalent to that of a healthy skeletal muscle in control subjects. Hence, making more capillaries may not be what is necessary to improve ischemic muscle perfusion, giving a potential explanation for the failure of angiogenic therapies. However, inflammation and interstitial edema suggested the implication of EC dysfunction. We then adapted a mouse model of chronic limb ischemia recapitulating the features of human CLI to test whether ameliorating EC function may improve muscle perfusion and decrease signs of CLI. We treated mice with the Hedgehog agonist SAG to improve EC function and found that it prevented muscle inflammation and edema, improved muscle perfusion and prevented myopathy thus revealing a new alternative therapy for CLI.
\end{abstract}

\section{Podosomes Mediate Basement Membrane Collagen-IV Proteolysis During Sprouting Angiogenesis}

\author{
Pirjo Spuul $^{1}$, Thomas Daubon ${ }^{1}$, Bettina Pitter $^{2}$, Florian Alonso ${ }^{* 1}$, Isabelle Fremaux ${ }^{1}$, \\ Eloi Montanez ${ }^{2}$, and Elisabeth Génot ${ }^{1}$ \\ ${ }^{1}$ Centre de recherche Cardio-Thoracique de Bordeaux [Bordeaux] (CRCTB) - Université Bordeaux \\ Segalen - Bordeaux 2, CHU Bordeaux [Bordeaux], Institut National de la Santé et de la Recherche \\ Médicale : U1045 - Université Bordeaux Segalen 146 rue Leo Saignat Zone sud, Batiment TP, 2ème \\ étage Case courrier 1333076 Bordeaux cedex, France \\ ${ }^{2}$ Walter-Brendel Center of Experimental Medicine, Ludwig-Maximilians University - Munich,
} Allemagne

Résumé

Introduction: During angiogenic sprouting, endothelial 'tip cells' emerge from existing vessels in response to VEGF-A. These cells are invasive meaning that they are capable of breaching the basement membrane that forms a sleeve around the capillary. Although it has been known for long that metalloproteases play a key role in this process, how cells handle this step have not been characterized. In the present work, we have examined whether $\mathrm{F}$-actin/cortactin/P-Src-based matrix-degrading microdomains called podosomes contribute to this step.

Methods: To explore the role of podosomes in the angiogenic process, we developed a novel asymmetric 3D-assay recapitulating sprouting angiogenesis in vitro. We used the mouse neonatal retinal angiogenesis model to detect then analyse podosome formation during sprouting angiogenesis in vivo.

Results: In vitro, VEGF-A/Notch signaling regulates the formation of functional podosomes in endothelial cells in 2D as well as in tip cell-like cells in 3D. In vivo, we demonstrate that tip cells assemble podosomes during physiological retinal angiogenesis. In the retina, podosomes are also part of an interconnected network that surrounds large microvessels and impinges on the underlying basement membrane. Consistently, collagen-IV is scarce in podosome areas. Moreover, Notch inhibition exacerbates podosome formation and collagen-IV loss, indicating that Notch signaling regulates this process.

Conclusion: We propose that the localized proteolytic action of podosomes on basement membrane collagen-IV facilitates endothelial cell sprouting and anastomosis within the developing vasculature. The identification of podosomes as key components of the sprouting machinery provides another opportunity to target angiogenesis therapeutically. 


\title{
How plant food bioactives may have benefits for vascular health? The case of curcumin.
}

\author{
Laurent-Emmanuel Monfoulet*1 ${ }^{1}$ Coban Dilek ${ }^{2}$, Sylvie Mercier ${ }^{1}$, Dominique Bayle ${ }^{1}$, \\ Nicolas Baber-Chamoux ${ }^{1,3}$, Dragan Milenkovic ${ }^{1}$, and Christine Morand ${ }^{1}$ \\ ${ }^{1}$ INRA, Unité de Nutrition Humaine- UMR 1019, Clermont-Ferrand, France. (UNH-UMR 1019 \\ INRA/UCA) — Institut national de la recherche agronomique (INRA) : UMR1019, Université Clermont \\ Auvergne - Unité de Nutrition Humaine - UMR 1019 Centre INRA Auvergne-Rhone-Alpes 63122 Saint \\ Genes Champanelle, France \\ ${ }^{2}$ INRA, Unite de Nutrition Humaine- UMR 1019, Clermont-Ferrand, France. (UNH-UMR 1019 \\ INRA/UCA) - Institut national de la recherche agronomique (INRA) : UMR1019, Université Clermont \\ Auvergne, Université Clermont Auvergne - Unité de Nutrition Humaine - UMR 1019 Centre INRA \\ Auvergne-Rhone-Alpes 63122 Saint Genes Champanelle, France \\ ${ }^{3} \mathrm{CHU}$ Gabriel Montpied (CHU) - CHU Clermont-Ferrand : Servicede Cardiologie — 58 rue \\ Montalembert 63000 Clermont-Ferrand, France \\ *Intervenant
}

Resume

Diet is recognized as an important determinant of health. During the last decade, the protective effect of fruits and vegetables on cardiovascular health has been correlated to the high content of these foods in bioactive compounds, especially polyphenols. The interest of these compounds for cardiovascular health is also supported by clinical studies reporting beneficial effects of polyphenol-rich fruit consumption on intermediate markers of cardiovascular diseases. In addition, animal and in vitro studies provided a number of results accounting for a positive role of polyphenols to prevent some dysfunctions associated to the development of cardiovascular diseases. Among these bioactives is curcumin. We previously showed that curcumin reduced the infiltration of immune cells into the vascular wall and prevented atherosclerosis development in mice. This study aimed to decipher the underlying mechanisms of these actions investigating the effect of curcumin on endothelial cell function. Human umbilical vein endothelial cells (HUVECs) were exposed to curcumin $(0.5-1 \mu \mathrm{M})$ for 3 hours prior to their activation by Tumor Necrosis Factor alpha (TNF-a). Endothelial permeability, monocyte adhesion and transendothelial migration assays were conducted under static condition and shear stress that mimics blood flow. We further investigated the impact of curcumin on signaling pathways and on the expression of genes using macroarrays.

Pre-exposure of endothelial cells to curcumin reduced monocyte adhesion and their transendothe-

lial migration in both static and shear stress conditions. Curcumin also prevented changes in both endothelial permeability and the area of HUVECs when induced by TNF-a. We showed that curcumin modulated the expression of 15 genes involved in the control of cytoskeleton and endothelial junction dynamic. Our findings demonstrate the ability of curcumin to reduce monocyte TEM through a multimodal regulation of the endothelial cell dynamics with a potential benefit on the vascular endothelial function barrier.

Mots-Clés: polyphenols, curcumin, vascular endothelial cells, integrity 
Abstract List

P.01 STUDYING IN VIVO THE INFLUENCE OF ISOMETRIC CONTRACTION IN HUMAN VASCULAR PHYSIOLOGY. Margarida Florindo, Henrique Silva and Luis Monteiro Rodrigues

P.02 EXPLORING THE MURINE MICROVASCULAR RESPONSE TO HYPEROXIA WITH THE WAVELET TRANSFORM. Henrique Silva, Alain-Pierre Gadeaux and Luis Monteiro Rodrigues

P.03 ABOUT THE LASER DOPPLER FLOWMETRY SIGNAL OSCILLATORY PROPERTIES OF A MURINE MODELS OF DIABETES. Hentique Silva, Alain-Pierre Gadeaux and Luis Monteiro Rodrigues.

P.04 MICROCIRCULATORY RESPONSE TO IN VIVO MASSAGE IN THE LOWER LIMB. Clemente Rocha, Henrique Silva, Hugo Ferreira and Luis Monteiro Rodrigues

P.05 CALCULATION OF CENTRAL BLOOD PRESSURE BY ANALYZING THE CONTOUR OF THE PHOTOPLETHYSMOGRAPHIC PULSE MEASURED AT THE FINGER WITH THE POPMÈTRER DEVICE. Hasan Obeid

P.06 CLINICAL EVIDENCE OF THE VASCULAR PROTECTIVE EFFECTS OF GRAPEFRUIT FLAVANONES IN POST-MENOPAUSAL WOMEN AND POTENTIAL MOLECULAR MECHANISMS INVOLVED. Dragan Milenkovic, Véronique Habauzit, Laurent-Emmanuel Monfoulet, Marie-Anne Verny, Dominique Bayle, Céline Boby, Nicolas Barber-Chamoux, André Mazur, Claude Dubray and Christine Morand. ANTHOCYANINS AND THEIR GUT METABOLITES REDUCE MONOCYTE ADHESION AND MIGRATION ACROSS TNFALPHA-ACTIVATED ENDOTHELIAL CELLS TROUGH NUTRIGENOMIC MECHANISMS REGULATING ENDOTHELIAL PERMEABILITY. Irena Krga, Radu Tamaian, Sylvie Mercier, Céline Boby, Laurent-Emmanuel Monfoulet, Christine Morand and Dragan Milenkovic. THE ROLE OF THE BLOOD-BRAIN BARRIER IN AMYLOID-B BIOLOGY: CONTRIBUTION TO ALZHEIMER'S DISEASE. Amaia Dominguez-Belloso

P.09 MANIPULABLE AND PERFUSABLE ARTIFICIAL BLOOD VESSELS PRODUCED BY 3D VASCULAR TISSUE ENGINEERING. Laetitia Andrique, Gaelle Recher, Kevin Alessandri, Maxime Feyeux, Pierre Nassoy and Andréas Bikfalvi. 


\section{Studying in vivo the influence of isometric contraction in human vascular physiology}

Margarida Florindo ${ }^{* 1,2}$, Henrique Silva ${ }^{2,3}$, and Luis Monteiro Rodrigues ${ }^{\dagger 2,3}$

${ }^{1}$ Department of Physiotherapy, Portuguese Red Cross Health School (ESCSCVP) - Avenida de Ceuta, 1 Edifício Urbiceuta, 1300-125 Lisboa, Portugal

${ }^{2}$ Universidade Lusófona's Research Center for Biosciences and Health Technologies (CBIOS) - Campo Grande 376, 1740-024 Lisboa, Portugal

${ }^{3}$ Pharmacol. Sc Depart - Universidade de Lisboa, Faculty of Pharmacy - Av. Prof. Gama Pinto, 1649-003 Lisboa, Portugal

\section{About the laser Doppler flowmetry signal oscillatory properties of a murine models of diabetes}

Henrique Silva ${ }^{1,2}$, Alain-Pierre Gadeau ${ }^{3}$, and Luis Monteiro Rodrigues*1,2

${ }^{1}$ Universidade Lusófona's Research Center for Biosciences and Health Technologies (CBIOS) - Campo Grande 376, 1740-024 Lisboa, Portugal

${ }^{2}$ Pharmacol. Sc Depart — Universidade de Lisboa, Faculty of Pharmacy - Av. Prof. Gama Pinto, 1649-003 Lisboa, Portugal

${ }^{3}$ Université de Bordeaux INSERM, U1034, Adaptation cardiovasculaire à l'ischémie - Université de Bordeaux (Bordeaux, France) - 1 Av. Magellan, 33600 Pessac, France

\section{Résumé}

The so called "muscular pump" is a major determinant of the circulatory homeostasis, in particular in the lower limb as part of the venous return mechanisms. However, the mutual relationships established between the muscle under activity and the local circulation, is not entirely characterized. So, addressing these aspects, here we develop an experimental model to evaluate the impact of the gastrocnemius activity on the microcirculation. Six healthy subjects ( $31+9$ years old) both genders (3 female and 3 male) participated in this study after informed consent. We tested the isometric plantar flection in the standing position. After standing for $5 \mathrm{~min}$, subjects maintained for $1 \mathrm{~min}$ an isometric plantar flexion with both feet, then resuming the original position for another $5 \mathrm{~min}$. Local (foot) perfusion was evaluated by photoplethysmography (PPG) and laser Doppler flowmetry (LDF). Pulse rate (PR) was obtained from PPG and the calf muscle activity followed by electromyography (EMG). Nonparametric statistics were used for phase comparisons. During the gastrocnemius activity significant perfusion changes were detected for LDF and PPG signals, rapidly returning to basal values after contraction stopped. The pulse rate did not change during the three protocol phases. Thus, this model looks suitable to further explore the the impact of muscle contraction in the human in vivo microcirculation.

Mots-Clés: gastrocnemius, microcirculation, EMG, LDF, PPG

$$
\begin{aligned}
& \text { *Auteur correspondant: mflorindo@esscvp.eu } \\
& { }^{\dagger} \text { Intervenant }
\end{aligned}
$$

sciencesconf.org:viva-liac2017:175162

\section{Exploring the murine microvascular response to} hyperoxia with the wavelet transform

\footnotetext{
Henrique Silva ${ }^{* 1,2}$, Alain-Pierre Gadeau ${ }^{3}$, and Luis Monteiro Rodrigues ${ }^{* 1,2}$
}

${ }^{1}$ Universidade Lusófona's Research Center for Biosciences and Health Technologies (CBIOS) - Campo Grande 376, 1740-024 Lisboa, Portugal

${ }^{2}$ Pharmacol. Sc Depart - Universidade de Lisboa, Faculty of Pharmacy - Av. Prof. Gama Pinto, 1649-003 Lisboa, Portugal

${ }^{3}$ Université de Bordeaux INSERM, U1034, Adaptation cardiovasculaire à l'ischémie - Université de

Bordeaux (Bordeaux, France) - 1 Av. Magellan, 33600 Pessac, France, France
Résumé

Skin microcirculation is widely regarded as a representative model to study physiological and pathophysiological vascular regulation mechanisms. Laser Doppler flowmetry (LDF) and pathophysiological vascular regulation mechanisms. Laser Doppler flowmetry (LDF)
signal, although its complex oscillatory origin, has been very helpful in understanding misignal, although its complex oscillatory origin, has been very helpful in understanding mi-
crovascular dynamics in vivo. Hyperoxia is also used as a stimulus that accentuates the differences in microvascular responsiveness between normal and pathological states, helping to test the dy to test these dynamics. Here, we aimed to characterize the microvascular reactivity of niming groups wo stic \pm 9.1 woks sisn) were ery) under ketamine-xylazine anesthesia. All procedures involving animal experimentatio wer termine the pympathetic, NO endothelial dep of the LD (DFA) a the etropy pof (DFA) and the entropy profle was given by the complexity index from the multiscaled en-

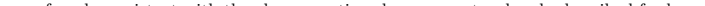
were fornd, chistent win the above montion WT annificant differences were for WFA analys des DFA revealed significant differences in the fractal organization of the myogenic and sympathetic LDE cop myogenic and symathetic activties mib/d useful in distinguishing between physiological and pathophysiological microvascular profiles. In this study, our results

Mots-Clés: Diabetes, LDF, wavelet transform, DFA, MSE

*Intervenant

sciencesconf.org:viva-liac2017:175164

Microcirculatory response to in vivo massage in the lower limb

Clemente Rocha ${ }^{* 1}$, Henrique Silva ${ }^{1,2}$, Hugo Ferreira ${ }^{3}$, and Luis Monteiro Rodrigues ${ }^{\dagger 1,2}$

Universidade Lusófona's Research Center for Biosciences and Health Technologies (CBIOS) - Campo Grande 376, 1740-024 Lisboa, Portugal

${ }^{2}$ Pharmacol. Sc Depart - Universidade de Lisboa, Faculty of Pharmacy - Av. Prof. Gama Pinto, 1649-003 Lisboa, Portugal

${ }^{3}$ Institute of Biophysics and Biomedical Engineering, Universidade de Lisboa, Faculty of Sciences Campo Grande 016, 1749-016 Lisboa, Portugal

\section{Résumé}

Massage is a common procedure, also used with therapeutical purposes in the lower limb to alleviate some of the symptom related with peripheral vascular disease. Its effects are still poorly understood., so we developed a protocol to characterize in vivo, the cutaneous microcirculation response measured by Laser Doppler flowmetry (LDF) and photoplethys-
mography (PPG) to mase 16 young belthy subjects (19.6 1.3 y.) both gender, were subjected to two therapeutical massage (TM) protocols, one applied in the upward direction (upM) and the other downwards (dwM), each consisting of three phases - $10 \mathrm{~min}$ baseline (phase I), 5 min massage (phase II) and $10 \mathrm{~min}$ recovery (phase III). One random lower limb was massaged (test), while the contralateral served as control. Blood flow was measured on the first and second toes of both feet with PPG and LDF, respectively. The wavelet transform (WT) analysis was applied to decompose these signals into their main activity components (cardiac, respiratory, myogenic, sympathetic, endothelial NO-dependent (NOd) and endo $(\mathrm{p}<0.05)$ - During both protocols, both PPG and LDF registered a significant perfusion increase in both feet. After decomposition, significant changes in the signals' components were also detected, for both feet in all cases. For the upM protocol, both techniques recorded a significant increase in the respiratory activity for both feet. LDF also recorded a significant increase in the cardiac and a decrease in the NOd activities, while PPG recorded a significant increase in the myogenic and a decrease in the NOi activities. For the dwM protocol, both techniques recorded a significant increase in the respiratory and myogenic activities, together with a significant decrease of the NOi activity. As for LDF, a significant increase in the cardiac activity was noted, whereas for PPG a significant decrease in the NOd was noted. These results suggest that, under these conditions, massage modifies the microvascular reactivity increasing the lower limb perfusion, although different mechanisms in similar principles might register different features at different depths.

Mots-Clés: Massage, lower limb, vascular regulation, LDF, PPG

${ }^{*}$ Auteur correspondant: c.rocha.dr@gmail.com 
Calculation of central blood pressure by analyzing the contour of the photoplethysmographic pulse measured at the finger with the pOpmètre $R$ device

Hasan Obeid $^{* 1}$

${ }^{1}$ Inserm - Université Paris V - Paris Descartes - 56 rue leblanc, Paris, France

\section{Résumé}

Calculation of central blood pressure by analyzing the contour of the photoplethysmographic pulse measured at the finger with the pOpmètre® device Background: Analysis of the contour of the peripheral pulse to assess arterial properties was first described in the nineteenth century. The reference technique to estimate central blood pressure (CBP), non-invasively, is by analyzing the radial pressure pulse acquired using a tonometer and then to establish a transfer function relating the radial pressure to the CBP. An alternative technique utilizes a volume pulse. This may conveniently be acquired optically from a finger (digital volume pulse obtained with the photodiode of the pOpmètre $囚$ device). Although less widely used, this technique deserves further consideration because of its simplicity and ease of use.

Objectives: The objective is to establish a transfer function estimating the central blood pressure $(\mathrm{CBP})$, calibrated with a brachial pressure cuff and using the parameters obtained with the contour analysis of the photoplethysmographic pulse measured at the finger with the pOpmètre $\AA^{\text {system }}$

Methods: We positioned the photodiode sensor on the finger, insuring that the sensor's ens is in contact with the finger. Brachial blood pressure measurements was performed with a standard cuff. The central blood pressure values measured with Sphygmocor used as the reference values. Multiple regression analysis was done to establish the transfer functions. Pearson's correlation and Bland Altman graph were performed

Results: 69 subjects were included: 24 healthy subjects and 45 patients with essential hypertension aged $33 \pm 8$ years and $59 \pm 17$ years respectively. The correlation between the estimated contras systolic pressure (CSP) and the reference one, was good and significant $\left(\mathrm{r}^{2}\right.$ $=0.91 ; \mathrm{p}<0.0001)$. A better correlation was found in terms of central diastolic pressure (CDP) $\left({ }^{2}=0.92 ; \mathrm{p}<0.0001\right)$. The $\mathrm{Bl}$ ( (CDP) $\left(\mathrm{r}^{2}=0.92 ; \mathrm{p}<0.0001\right)$. The Bland and Altman analysis, mean difference was 4 $\mathrm{mmHg} \mathrm{p}<0.0001$ (CSP) versus $3 \mathrm{mmHg} \mathrm{p}<0.0001$ (CDP), the standard deviation of the difference was $5 \mathrm{mmHg}$ (CSP) versus $4 \mathrm{mmHg}$ (CDP), classifying the estimation as good
agreement.

Conclusions: The estimation of the central blood pressure with the finger pulse qualifies as good agreement with the reference technique for the central systolic and diastolic pressure estimation.

Mots-Clés: Arterial stiffness, Central Blood Pressure

sciencesconf.org:viva-liac2017:170950

The role of the blood-brain barrier in Amyloid- $\beta$ biology: contribution to Alzheimer's disease

Around $80 \%$ of Alzheimer's disease (AD) cases result in cerebral amyloid angiopathy (CAA) with amyloid- $\beta(\mathrm{A} \beta)$ deposition around blood vessels. The source of $\mathrm{A} \beta$ however is not entirely clear. Our group have previously shown that beta-secretase 1 (BACE-1), the key enzyme cleaving amyloid precursor protein (APP), is expressed by brain endothelial cells
(ECs). We therefore hypothesize that BACE-1 at the BBB might be involved in cleavage of
circulating APP and or neuronal APP, thus generating A $\beta$ locally at the BBB, reemphasizing circulating APP and or neuronal APP, thus generating $\mathrm{A} \beta$ locally at the BBB, reemphasizing the vascular aspect of $\mathrm{AD}$. In order to investigate the $\mathrm{BBB}$ contribution to $\mathrm{AD}$ formation and progression we made use of the Thy1-SwDI mouse model, harbouring triple mutation of amyloid precursor protein APP and being described to show onset of AD symptoms by 3 months of age. We could demonstrate that the AD mice have blood-brain barrier (BBB) dehis by changes in AD mice during ageing we teamed up with GenXPro to perform Massive AnalAD compared to aged-matched WT mice. We are expecting to identify genes regulated in MBMVs in $\mathrm{AD}$, which might shed novel light onto the $\mathrm{BBB}$ contribution to $\mathrm{AD}$ formation and progressio

Currently, we aim to further understand the role of BBB-dissuption for $\mathrm{AD}$ formation in our mouse model and the potential benefit of re-sealing the BBB. We have shown that Wnt/ $\beta$-catenin signalling contributes to BBB development and that dominant activatio of endothelial $\beta$-catenin signalling re-establishes barrier properties in brain tumour vessels. Therefore, we currently test the hypothesis that dominantly active $\beta$-catenin may slow dow $\mathrm{AD}$ progression. Preliminary data support a memory improvement in the $\mathrm{AD}$ mouse mode upon inducible $\beta$-catenin signalling in $\mathrm{ECs}$, indicated by $\mathrm{Y}$-maze behavioural tests for learning and memory. Morever, we further investigate BACE-1 at the BBB for AD. Hereis ageing.
Together, our results point into a novel contribution of $\mathrm{ECs}$ to $\mathrm{CAA}$ characteristic in $\mathrm{AD}$, as well as to a therapeutic potential of the canonical Wnt pathway in $\mathrm{AD}$.

Mots-Clés: Alzheimer, blood, brain barrier, $\mathrm{AD}, \mathrm{BBB}, \mathrm{BACE}, 1$
Clinical evidence of the vascular protective effects of grapefruit flavanones in post-menopausal women and potential molecular mechanisms involved

Dragan Milenkovic ${ }^{1}$, Véronique Habauzit ${ }^{1,2}$, Laurent-Emmanuel Monfoulet ${ }^{* 1}$, Marie-Anne Verny ${ }^{1}$, Dominique Bayle ${ }^{1}$, Céline Boby ${ }^{3}$, Nicolas Baber-Chamoux ${ }^{1,4}$, André Mazur $^{1}$, Claude Dubray ${ }^{2}$, and Christine Morand ${ }^{1}$

${ }^{1}$ INRA, Unité de Nutrition Humaine- UMR 1019, Clermont-Ferrand, France. (UNH-UMR 1019 INRA/UCA) - Institut national de la recherche agronomique (INRA) : UMR1019, Université Clermont Auvergne - Unité de Nutrition Humaine - UMR 1019 Centre INRA Auvergne-Rhône-Alpes 63122 Saint Genès Champanelle, France

${ }^{2}$ CIC Clermont Ferrand - Institut National de la Santé et de la Recherche Médicale : CIC1405, Centre de Pharmacologie Clinique, CHU Gabriel-Montpied - Faculté de Médecine - 58 Rue Montalembert 63000 Clermont-Ferrand, France

${ }^{3}$ Unité Mixte de Recherches sur les Herbivores (UMRH) - Institut National de la Recherche

Agronomique : UMR1213, VetAgro Sup : UMR1213 - INRA / Site de Theix / 63122 Saint-Genès-Champanelle, France

${ }^{4} \mathrm{CHU}$ clermont Ferrand (Service de Cardiologie) - CHU Gabriel Montpied [Clermont-Ferrand] : Servicede Cardiologie - CHU Clermont Ferrand Hopital Gabriel Montpied 58, rue Montalembert 63003 Clermont-Ferrand Cedex 1, France

\section{Résumé}

Epidemiological studies reported that a high flavanone intake is associated with a reduced risk of cardiovascular diseases (Mink et al, 2007; Cassidy et al, 2012), however clinical evidence is still lacking. We carried out a cross-over RCT on 52 healthy post-menopausal women who have to consumed daily and for 6 -month, $340 \mathrm{ml}$ of grapefruit juice (providing $212 \mathrm{mg}$ naringenin-glycosides) or of an iso-energetic control beverage mimicking the compo sition of the juice but without naringenin. The aim of this trial was to evaluate the impact of GFJ consumption (i) on vascular function (blood pressure, endothelial function and arterial stiffness) and (ii) on the gene and miRNA expression in PBMCs isolated from enrolled volunteers using microarrays.

The intervention with GFJ improved pulse wave velocity (PWV), an indicator of arterial stiffness, without affecting endothelial function (FMD) or blood pressure. The nutrigenomic study showed that the regular intake of naringenin through GFJ consumption modul ted tudy showed that the regular intake of naringenin through GFJ consumption modulate the expression of genes and miRNAs in PBMCs. Bioinformatic analysis of microarray dat revealed that the are involved in different cellular processes, including inflammatory processes, chemotaxis, and circulating immune cells. The observed changes in genes and miRNAS expression profiles suggest a lower adhesion and infiltration of immune cells into the vascular wall. This

*Intervenant

sciencesconf.org:viva-liac2017:166826

Anthocyanins and their gut metabolites reduce monocyte adhesion and migration across TNFalpha-activated endothelial cells trough nutrigenomic mechanisms regulating endothelial permeability

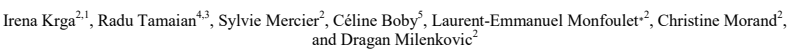

${ }^{2}$ INRA, Unité de Nutrition Humaine- UMR 1019, Clermont-Ferrand, France. (UNH-UMR 1019 INRA/UCA) - Institut national de la recherche agronomique (INRA) : UMR1019, Universit'e Clermont Auvergne - Unité de Nutrition

Humaine - UMR 1019 Centre INRA Auvergne-Rh^one-Alpes 63122 Saint Gen'es Champanelle, France ${ }^{1}$ University of Belgrade - Centre of Research Excellence in Nutrition and Metabolism, Institute for Medical Research, University of Belgrade, Serbia, Serbie

${ }^{4}$ SC Biotech Corp SRL - SC Biotech Corp SRL, Romania, Roumanie 3National Institute for Research and Development for Cryogenic and Isotopic Technologies - National Institute for Research and Development for Cryogenic and Isotopic Technologies, Romania;, Roumanie ${ }^{5}$ Unité Mixte de Recherches sur les Herbivores (UMRH) - Institu National de la Recherche Agronomique : UMR1213, VetAgro Sup : UMR1213 - INRA / Site de Theix / 63122 SaintGenès-Champanelle, France

$*$ Intervenant

Résumé

Cardioprotective effects of dietary anthocyanins are partly attributed to their ability to maintain endothelial function However, the underlying mechanisms of action are not fully understood. This study aimed to test the effect of anthocyanins and their gut metabolites, at physiologically-relevant conditions, on endothelial cell function and decipher the underlying molecular mechanisms of action using integrated omics approaches. Primary endothelial cells were treated with a mix of $0.1 \mathrm{mM}$ cyanidin-3-arabinoside, $0.1 \mathrm{mM}$ cyanidin-3-galactoside, $0.1 \mathrm{mM}$ cyanidin-3 were treted wh a mix of $0.1 \mathrm{mM}$ cyanidin-3-arabinoside, $0.1 \mathrm{mM}$ cyanid -3-galactoside, $0.1 \mathrm{mM}$ cyanidin-3glucoside, $0.1 \mathrm{mM}$ delphinidin-3-glucoside, $0.1 \mathrm{mM}$ peonidin-3-glucoside and $0.5 \mathrm{mM}$ 4-hydroxybenzaldehyde for $3 \mathrm{H}$ or with a mix of gut metabolites. $0.2 \mathrm{mM}$ protocatechuic, $2 \mathrm{mM}$ vanillic, $1 \mathrm{mM}$. Additionaly, succis following the transmigration. Gene and miRNA expression, signaling protein phosphorylation and molecular docking were transmigration.

All mixes significantly reduced monocyte adhesion and transendothelial migration by $24.5 \%$ and $46.2 \%$ in average. Gene expression analysis using TLDA arrays showed that mixtures modulated the expression of genes involved in cell-cell adhesion, cytoskeleton organization or focal adhesion. Bioinformatic analyses were done to identify potential transcription factors involved in the observed nutrigenomic effects and signalling proteins regulating their activity. Western blot analysis showed the ability of tested compounds to modulate phosphorylation of NfkB-p65 or p38 signalling proteins. Docking identified 25 out of 63 potential cell signaling proteins to which these compounds can bind to and initiate the observed nutrigenomic effect. The mixes also affected miRNA expression. Bioinformatic analysis showed that more than half of modulated miRNAs are involved in regulation of endothelial cell permeability, contributing to the observed changes in endothelial cell function. Integration of these results revealed endothelialprotective properties of anthocyanins and their gut metabolites and deciphered new underlying multi-target and multilayered mode of action 


\title{
Manipulable and perfusable artificial blood vessels produced by $3 \mathrm{~d}$ vascular tissue engineering
}

\author{
Laetitia ANDRIQUE $^{* 1,2}$, Gaelle RECHER ${ }^{2}$, Kevin ALESSANDRI ${ }^{2}$, Maxime FEYEUX ${ }^{2}$ \\ Pierre NASSOY ${ }^{2}$ and Andréas BIKFALVI \\ ${ }^{1}$ INSERM U1029 Laboratory of Angiogenesis and Cancer Microenvironment, Talence, FRANCE \\ ${ }^{2}$ UMR 5298 LP2N Institut d'Optique Graduate School, Talence, FRANCE \\ Today, vasculature-related improvement is a key question in clinics since cardio-vascular \\ diseases are the first cause of premature death globally (according to World Health Organization, \\ WHO). In addition, tissue engineering is limited in constructing functional organs essentially due to \\ the lack of vascularization to provide nutrients. Thus, to achieve the production of thick tissues, \\ vasculature is needed and it is crucial to develop strategies for in vitro blood vessel engineering.
}

Here, we propose a novel blood vessel engineering approach, which consist in a coencapsulation of ECs (HUVEC), SMC, and ECM in an alginate tubular shell. With this approach, we achieved the production of artificial mature blood vessels that are 1/ manipulable, 2/ histologically relevant, 3 / with variable inner and outer diameter, 4 / perfusable, 5/ tight and 6/ contractile. Moreover, this technique circumvents the actual limitations that exist for synthesis of small diameter artificial blood vessels $(<2 \mathrm{~mm})$.

Finally, this vascular tissue engineering approach can serve as a model for angiogenesis studies (in vitro, or following engraftments), cardiovascular drug testing (high-content screening in 3D tissues), tissue-engineered organoids vascularization and vascular grafts (surgical replacement of damaged tissues). 